\title{
Exact Spatiotemporal Dynamics of Confined Lattice Random Walks in Arbitrary Dimensions: A Century after Smoluchowski and Pólya
}

\author{
Luca Giuggioli॰ \\ Bristol Centre for Complexity Sciences and Department of Engineering Mathematics, \\ University of Bristol, Bristol, BS8 1UB, United Kingdom
}

(Received 30 January 2020; revised manuscript received 19 March 2020; accepted 31 March 2020; published 28 May 2020)

\begin{abstract}
A lattice random walk is a mathematical representation of movement through random steps on a lattice at discrete times. It is commonly referred to as Pólya's walk when the steps occur in either of the nearestneighbor sites. Since Smoluchowski's 1906 derivation of the spatiotemporal dependence of the walk occupation probability in an unbounded one-dimensional lattice, discrete random walks and their continuous counterpart, Brownian walks, have developed over the course of a century into a vast and versatile area of knowledge. Lattice random walks are now routinely employed to study stochastic processes across scales, dimensions, and disciplines, from the one-dimensional search of proteins along a DNA strand and the two-dimensional roaming of bacteria in a petri dish, to the three-dimensional motion of macromolecules inside cells and the spatial coverage of multiple robots in a disaster area. In these realistic scenarios, when the randomly moving object is constrained to remain within a finite domain, confined lattice random walks represent a powerful modeling tool. Somewhat surprisingly, and differently from Brownian walks, the spatiotemporal dependence of the confined lattice walk probability has been accessible mainly via computational techniques, and finding its analytic description has remained an open problem. Making use of a set of analytic combinatorics identities with Chebyshev polynomials, I develop a hierarchical dimensionality reduction to find the exact space and time dependence of the occupation probability for confined Pólya's walks in arbitrary dimensions with reflective, periodic, absorbing, and mixed (reflective and absorbing) boundary conditions along each direction. The probability expressions allow one to construct the time dependence of derived quantities, explicitly in one dimension and via an integration in higher dimensions, such as the first-passage probability to a single target, return probability, average number of distinct sites visited, and absorption probability with imperfect traps. Exact mean firstpassage time formulas to a single target in arbitrary dimensions are also presented. These formulas allow one to extend the so-called discrete pseudo-Green function formalism, employed to determine analytically mean first-passage time, with reflecting and periodic boundaries, and a wealth of other related quantities, to arbitrary dimensions. For multiple targets, I introduce a procedure to construct the time dependence of the first-passage probability to one of many targets. Reduction of the occupation probability expressions to the continuous time limit, the so-called continuous time random walk, and to the space-time continuous limit is also presented.
\end{abstract}

DOI: 10.1103/PhysRevX.10.021045

Subject Areas: Statistical Physics

\section{INTRODUCTION}

While random walks have their root in the 17th century analysis of games of chance [1], they are associated with Pearson who introduced them in 1905 [2], although many of their properties had already been elucidated in 1900 by Bachelier [3] whose work remained largely unknown until the 1950s [4]. It was Smoluchowski, in 1906 [5], who first

Published by the American Physical Society under the terms of the Creative Commons Attribution 4.0 International license. Further distribution of this work must maintain attribution to the author(s) and the published article's title, journal citation, and DOI. derived the relation between diffusion and lattice random walks (LRW), that is, walks in discrete space and time, laying the foundations of the modern theory of stochastic processes and Brownian motion [6-12] (see also Ref. [13] for a more detailed historical account of the relevant scientists and their contributions to the kinetic theory of matter in the early part of last century). In mathematics, LRW are a special class of Markov chains [14]; they were popularized in the 1920s by Pólya's seminal work $[15,16]$ on the dimensionality dependence of the recurrence probability, that is, the probability that a random walker on an infinite space lattice eventually returns to its starting point.

With advances in chemistry and material sciences in the 1950s and 1960s, a theoretical need to characterize particle 
or excitation transport in disordered materials such as amorphous semiconductors and molecular aggregates emerged $[17,18]$. Theoretical techniques to estimate reaction diffusion processes in such materials were the focus of LRW research [19-23] and have continued since then [24-26].

The diffusive paradigm soon crossed disciplinary boundaries and pervaded other scientific disciplines. Researchers began using random walk models, both discrete and continuous, in life sciences to represent the movement of organisms in animal ecology [27-31] and cell biology [32-36] and later in social sciences, e.g., in social network analysis [37] and financial systems [38]. The incredible level of sophistication and reach of random walks have made them become de facto the null model to interpret a variety of observations across many disciplines.

In physics, LRW have been extensively employed to study anomalous diffusive processes $[39,40]$, self-avoiding walks [41], critical phenomena [42], and search processes [43-46] and, more recently, excluded volume interactions $[47,48]$, fractional dynamics [49], record statistics [50,51], and non-Markov processes [52,53].

In mathematics, from being instrumental to the development of probability theory in the early part of the last century [54], LRW have grown to encompass a large body of work that draws from symbolic enumeration methods and complex analysis to study fundamental structures such as permutations, sequences, trees, and graphs [55-57]. Nowadays, LRW play a fundamental role in analytic combinatorics [58], with wide ranging applications to areas such as actuarial sciences [59], queuing theory, branching processes, and dynamic data structure [60].

In comparison to processes in continuous space and time, the discreteness of LRW has two theoretical advantages. In the presence of spatial heterogeneities, the use of a lattice, rather than a continuous space variable, avoids the need to solve boundary value problems. Discrete time, resulting, e.g., from regular sampling of certain observables, facilitates the determination of time-dependent quantities as no process may occur with an infinite number of steps in a very small time interval. However, the convenience of spatiotemporal discreteness is counteracted by the notorious difficulty of finding closed-formed expressions in transport calculations [43] when compared to discrete space and continuous time as well as to continuous space and continuous time formulations.

This difficulty is particularly evident when seeking analytic formulas to represent the LRW occupation probability, the so-called Green's function or propagator, in bounded domains. While for unbounded domains the spatiotemporal one-dimensional (1D) propagator, derived by Smoluchowski [5,61], as well as its generating function (discrete Laplace transform) in arbitrary dimensions [19], are well known, for finite domains, only a few exact results exist.

Confidence in the ability to analytically find the time dependence of confined Pólya's walk propagators stems from the intimate connection $[63,64]$ between the one-step transition matrix and orthogonal polynomials. However, over the years, it has become apparent that finding such polynomials explicitly can be a challenge [65], in particular, for dimensions larger than one.

For 1D confined domains, the time-dependent propagator for absorbing boundaries [54] and periodic domains [66] have been known for a long time. But for reflecting boundaries, i.e., a 1D box, and with one absorbing and one reflecting boundary, exact propagators have not appeared in the literature. A recent attempt to approximate the fully reflecting case has exploited the known propagator in a periodic domain and the analogy of the geometry of a box to that of a squeezed ring [67].

In higher dimensions, propagators in closed form exist only for periodic domains [66]. No analytic results are known for absorbing domains, reflecting domains, or mixed reflecting and absorbing domains. But more importantly, a procedure to derive exact propagator expressions in arbitrary dimensions with any combination of absorbing, periodic, reflecting, or mixed boundaries is lacking.

Here, I bridge this knowledge gap by developing a procedure to construct, for arbitrary dimensions, the analytic propagator in the time domain as well as its generating function. I derive various time-dependent quantities associated with the propagator, such as the first-passage probability to one and multiple targets, the average number of distinct sites visited, the return probability, and the absorption probability at a partially absorbing trap. Furthermore, I employ the propagators' analytic expressions to determine the mean first-passage time (MFPT) to a set of targets in arbitrary dimensions as a function of the elementary MFPT to single targets.

The content of the paper is organized as follows. Section II treats the derivation of the $1 \mathrm{D}$ propagator and its generating function in the presence of different boundary conditions, pictorially represented in Fig. 1. The analytic time dependence of quantities derived from the 1D propagators, such as the first-passage probability, the return probability, and the average number of distinct sites visited, is shown in Sec. III, whereas 1D MFPT and mean exit time expressions are presented in Sec. IV. Section V gives the dimensionality reduction to obtain the spatiotemporal dependence of LRW propagators in arbitrary dimensions and arbitrary boundary conditions. The recovery of known propagators in continuous time and continuous space is also shown. In Sec. VI, I study the dynamics of partially absorbing traps, while the determination of MFPT to a single target in arbitrary dimensions, and its connection to the pseudo-Green function formalism, is treated in Sec. VII. In Sec. VIII, a formalism to construct the generating function of the first-passage probability and MFPT to any of a number of targets is presented. Concluding remarks and a discussion of the applicability of the analytic findings to other problems are given in Sec. IX. 


\section{TIME-DEPENDENT PROPAGATOR FOR THE SYMMETRIC RANDOM WALK IN ONE- DIMENSIONAL DOMAINS}

I start by considering the $1 \mathrm{D}$ dynamics of the so-called symmetric lazy random walker (see, e.g., Ref. [68]) in an unbounded domain. In this case, the transition probability is identical to those in the bulk of a finite domain (red lattice sites depicted in Fig. 1). The equation governing the evolution of the site occupation probability, $\mathcal{Q}(n, t)$, is given by

$$
\begin{aligned}
\mathcal{Q}(n, t+1)= & \frac{q}{2} \mathcal{Q}(n-1, t)+\frac{q}{2} \mathcal{Q}(n+1, t) \\
& +(1-q) \mathcal{Q}(n, t),
\end{aligned}
$$

with $n$ representing a lattice site on the line and $t$ being time. As shown later in Sec. V C, changes in the probability $q$ correspond to a linear rescaling of the rate of jumps between neighboring sites in continuous time and discrete space, and a linear rescaling of the diffusion constant for Brownian walks. The classical Pólya's walk, also called the
Bernoulli walk, corresponds to $q=1[69,70]$ and represents a walker that always moves when in the bulk.

It is straightforward to find (see the Appendix A) the generating function, or $z$ transform, $\tilde{\mathcal{Q}}_{n_{0}}(n, z)=$ $\sum_{t=0}^{\infty} \mathcal{Q}_{n_{0}}(n, t) z^{t}$, of Eq. (1) with the initial condition $\mathcal{Q}(n, 0)=\delta_{n, n_{0}}$, where $\delta_{\ell, \ell^{\prime}}$ is a Kroneker delta. From $\tilde{\mathcal{Q}}_{n_{0}}(n, z)$, the propagator in bounded space, $\tilde{P}_{n_{0}}(n, z)$, can be obtained by considering the contribution from each of the infinite number of images of the initial condition, which account for the multiple interactions of the walker with the boundaries $[5,9,24]$. With boundaries located at $n=1$ and $n=N$, the propagator takes the general form (see derivation in Appendix B)

$$
\tilde{P}_{n_{0}}^{(\gamma)}(n, z)=\frac{2 \mathcal{D}^{(\gamma)}\left[n, n_{0}, N, z\right]}{z q \sinh [\zeta(z)]},
$$

with $\cosh [\zeta(z)]=1+(1 / q)[(1 / z)-1], \quad \sinh [\zeta(z)]=$ $\sqrt{(1-z)[1-(1-2 q) z]} / q z$, and the symbols $\gamma=r, p$, $a$, and $m$ representing the various cases drawn in Fig. 1. The functional dependence of the function $\mathcal{D}^{(\gamma)}\left[n, n_{0}, N, z\right]$ is

$$
\mathcal{D}^{(\gamma)}\left[n, n_{0}, N, z\right]=\left\{\begin{array}{lc}
\frac{\cosh \left[\left(N-n_{>}+\frac{1}{2}\right) \zeta(z)\right] \cosh \left[\left(n_{<}-\frac{1}{2}\right) \zeta(z)\right]}{\sinh [N \zeta(z)]} & \gamma=r \\
\frac{\cosh \left[\left(N-\left|n-n_{0}\right|\right) \zeta(z)\right]+\cosh \left[\left|n-n_{0}\right| \zeta(z)\right]}{2 \sinh [N \zeta(z)]} & \gamma=p \\
\frac{\sinh \left[\left(N-n_{>}\right) \zeta(z)\right] \sinh \left[\left(n_{<}-1\right) \zeta(z)\right]}{\sinh [(N-1) \zeta(z)]} & \gamma=p \\
\frac{\cosh \left[\left(2 N-n_{>}-\frac{1}{2}\right) \zeta(z)\right] \cosh \left[\left(n_{<}-\frac{1}{2}\right) \zeta(z)\right]-\cosh \left[\left(n-\frac{1}{2}\right) \zeta(z)\right] \cosh \left[\left(n_{0}-\frac{1}{2}\right) \zeta(z)\right]}{\sinh [(2 N-1) \zeta(z)]} & \gamma=m,
\end{array}\right.
$$

where $n_{>}=\left(n+n_{0}+\left|n-n_{0}\right|\right) / 2$ and $n_{<}=\left(n+n_{0}-\right.$ $\left.\left|n-n_{0}\right|\right) / 2$. Note that for $\gamma=m$, the reflecting boundary is at $n=1$ [that is, $\tilde{P}_{n_{0}}(1, z)-\tilde{P}_{n_{0}}(0, z)=0$ ], whereas the absorbing boundary is at $n=N$ [that is, $\tilde{P}_{n_{0}}(N, z)=0$ ]. These 1D generating functions for the reflecting $(r)$, periodic $(p)$, and absorbing case ( $a$ ) for $q=1$ have appeared in the literature-respectively, in Refs. [54,66,71]—while the mixed boundary case $(m)$ appears to be unknown.

The $z$ inversion requires the complex integration of Eq. (2) via $f(t)=(2 \pi i)^{-1} \oint d z \tilde{f}(z) z^{-t-1}$ [72], with $|z|<1$ and with the integration contour being counterclockwise. As all poles of $\tilde{f}(z)$ are in the region $|z| \geq 1$, with the change of variable $z=1 / s$, one transforms the complex integral to $f(t)=(2 \pi i)^{-1} \oint d s \tilde{f}(1 / s) s^{t-1}$, with the contour in the region $|s|>1$ and with all poles now inside the contour $|s| \leq 1$. The integral is thus the sum of the residues at the poles located at $s=1, s=1-2 p$ and at $s_{k}^{(\gamma)}(q)=1-q+q \cos \left(\pi \mathcal{N}_{k}^{(\gamma)}\right), \quad k=1, \ldots, \omega^{(\gamma)}$,

with $\quad \mathcal{N}_{k}^{(r)}=k / N, \quad \mathcal{N}_{k}^{(p)}=2 k / N, \quad \mathcal{N}_{k}^{(a)}=k /(N-1)$, and $\mathcal{N}_{k}^{(m)}=(2 k-1) /(2 N-1)$ and with $\omega^{(r)}=\omega^{(p)}=$ $\omega^{(m)}=N-1$ and $\omega^{(a)}=N-2$. Evaluation of the residues then gives the corresponding time-dependent propagator

$$
P_{n_{0}}^{(\gamma)}(n, t)=\sum_{k=w^{(\gamma)}}^{\omega^{(\gamma)}} g_{k}^{(\gamma)}\left(n, n_{0}\right)\left[s_{k}^{(\gamma)}(q)\right]^{t},
$$

with $w^{(r)}=w^{(p)}=0$ and $w^{(a)}=w^{(m)}=1$ (note that $w^{(\gamma)}$ is the lower limit of the summation, while $\omega^{(\gamma)}$ is the upper limit), and with 


$$
g_{k}^{(\gamma)}\left(n, n_{0}\right)= \begin{cases}\frac{\alpha_{k} \cos \left[\left(n-\frac{1}{2}\right) \frac{\pi k}{N}\right] \cos \left[\left(n_{0}-\frac{1}{2}\right) \frac{\pi k}{N}\right]}{N} & \gamma=r \\ \frac{\cos \left[\left(n-n_{0}\right) \frac{2 \pi k}{N}\right]}{N} & \gamma=p \\ \frac{2 \sin \left(\frac{n-1}{N-1} k \pi\right) \sin \left(\frac{n_{0}-1}{N-1} k \pi\right)}{N-1} & \gamma=a \\ \frac{4 \cos \left[\left(n-\frac{1}{2}\right) \frac{2 k-1}{2 N-1} \pi\right] \cos \left[\left(n_{0}-\frac{1}{2}\right) \frac{2 k-1}{2 N-1} \pi\right]}{2 N-1} & \gamma=m,\end{cases}
$$

where $\alpha_{k}=1$ when $k=0$ and 2 for any $k>0$. As the generating function of Eq. (5) represents an alternative closed-form expression of $\tilde{P}_{n_{0}}^{(\gamma)}(n, z)$ in Eq. (2), it provides finite trigonometric series identities in the complex domain. Its generalizations, presented in Appendix E, are exploited extensively in deriving the propagators in higher dimensions.

While the time dependence for $q=1$ is already present in the literature for the absorbing (see, e.g., Ref. [54]) and

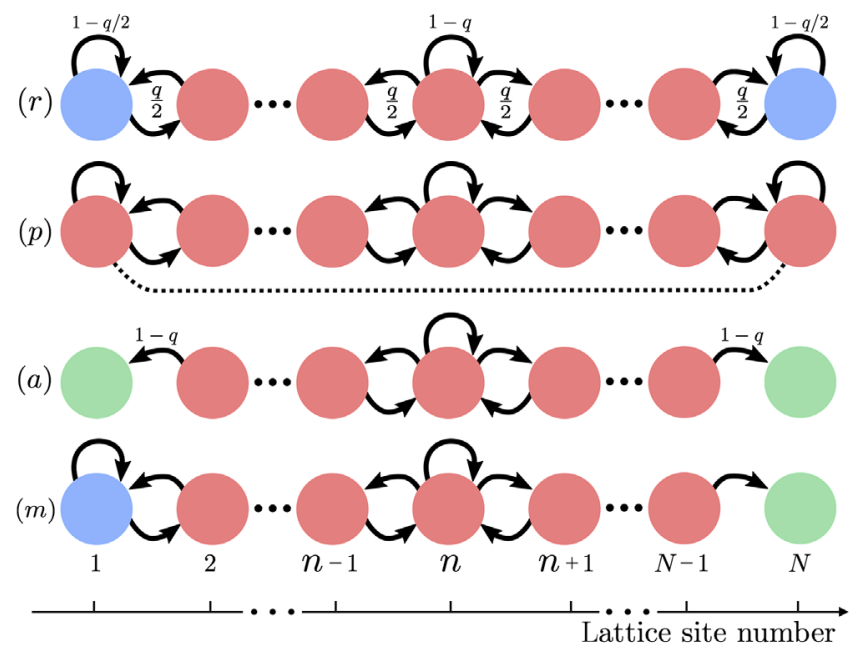

FIG. 1. Pictorial representation of the transition probabilities for a 1D LRW between neighboring lattice sites in the four boundary conditions studied, respectively, $(r)$ reflecting, $(p)$ periodic, $(a)$ absorbing, and $(m)$ mixed with one reflecting (left) and one absorbing (right). The boundaries are located at $n=1$ and $n=N$. Any red lattice site represents a lattice in the bulk of the domain for which the probability to move to a nearestneighbor site is $q / 2(0<q \leq 1)$ and the probability of staying in place is $1-q$. The boundary lattice sites are colored differently. At a reflecting site (blue circle), the probability of staying is $1-q / 2$, and $q / 2$ is the probability of moving towards the inside of the domain. In the translationally invariant periodic case, a dotted line connecting sites $n=1$ and $n=N$ to indicate equivalence between site 0 and site $N$ has been drawn. In such a case, all lattice sites are colored red as each one represents a site in the bulk of the domain. At an absorbing lattice point (green circle), only the transition probability $1-q$ into the site is possible. For clarity, the transition probabilities have been written only for a subset of the arrows in the figure. periodic cases (see, e.g., Ref. [66]), the other two cases appear to be unknown. Recently, for the case $q=1$, an alternative representation for the reflecting and absorbing cases has been proposed using the method of images with the unbounded space and time-dependent propagator [73], but its computability is limited given that it is expressed in terms of two infinite series.

An alternative procedure to find the exact propagator when $\gamma=r, a$, and $m$ is to solve the eigenvalue problem associated with the discrete diffusion equation

$$
P(n, t+1)=\sum_{\ell=1}^{N} \mathbb{A}_{n \ell} P(\ell, t),
$$

equivalently, $\vec{P}(t+1)=\mathbb{A} \cdot \vec{P}(t)$, in vectorial notation. Equation (7) describes the dynamics of the probability through the tridiagonal transition matrix $\mathbb{A}$. In the bulk, that is, for $n \neq 1, N$, Eq. (7) represents the same dynamics of the unbounded case in Eq. (1). The matrix $\mathbb{A}$ is such that $\mathbb{A}_{n m}=(1-q) \delta_{n, m}+(q / 2) \delta_{n, m+1}+(q / 2) \delta_{n, m-1}$, when $(n, m) \neq(1,1)$ or $(N, N)$, while the corner elements $\mathbb{A}_{11}$ and $\mathbb{A}_{N N}$ change depending on the boundary conditions. For the reflecting case, $\mathbb{A}_{11}=\mathbb{A}_{N N}=1-q / 2$, and for the absorbing case, $\mathbb{A}_{11}=\mathbb{A}_{N N}=1-q$ (that is, the diagonal elements of the matrix are all identical); when the reflection is at $n=1$ and the absorption is at $n=N$, one has $\mathbb{A}_{11}=$ $1-q / 2$ and $\mathbb{A}_{N N}=1-q$.

Note that $\vec{P}(t)$ in Eq. (7) can be iteratively constructed from the initial condition as $\vec{P}(t)=\mathbb{A}^{t} \cdot \vec{P}(0)=\mathbb{E} \mathbb{\natural}^{t} \mathbb{E}^{-1} \vec{P}(0)$, where $\mathbb{H}, \mathbb{E}$, and $\mathbb{E}^{-1}$ represent, respectively, the diagonal matrix of eigenvalues, and the matrix of the left and the right eigenvectors. As matrix $\mathbb{A}$ is a special tridiagonal Toeplitz matrix with two perturbed corners, its eigenvalues and eigenvectors can be determined exactly [74], leading to Eq. (5) for $\gamma=r, a$, and $m$.

\section{TIME DEPENDENCE OF RETURN PROBABILITY, FIRST-PASSAGE PROBABILITY, AND MEAN NUMBER OF DISTINCT VISITED SITES IN ONE DIMENSION}

The first time an event occurs is often an important feature of a stochastic system. The event might be represented 
by a random walker reaching a specific location on the lattice or returning to the starting location. The probabilities associated with the occurrence of these two events are called, respectively, first-passage probability and return probability. Their dynamics is linked to the one of the propagator via the renewal equation (see, e.g., Ref. [19]), $P_{\vec{n}_{0}}(\vec{n}, t)=\delta_{t, 0} \delta_{\vec{n}, \vec{n}_{0}}+\sum_{s=0}^{t} F_{\vec{n}_{0}}(\vec{n}, s) P_{\vec{n}}(\vec{n}, t-s)$, which has been represented in vectorial notation given its general validity. The equation links the propagator $P_{\vec{n}_{0}}(\vec{n}, t)$ to the first-passage probability $F_{\vec{n}_{0}}(\vec{n}, t)$ to reach $\vec{n}$ for the first time at time $t$ starting from $\vec{n}_{0}$, and to the return probability $R_{\vec{n}}(t)$ when $\vec{n}=\vec{n}_{0}$. It provides mathematical relations between the generating function of the return probability or the first-passage probability and the propagator. The first-return probability to site $\vec{n}$ is related via $\tilde{R}_{\vec{n}}(z)=$ $1-1 / \tilde{P}_{\vec{n}}(\vec{n}, z)$, while the first-passage probability is related via $\tilde{F}_{\vec{n}_{0}}(\vec{n}, z)=\tilde{P}_{\vec{n}_{0}}(\vec{n}, z) / \tilde{P}_{\vec{n}}(\vec{n}, z)$. Both relations are obtained by $z$ transforming the renewal equation.

For 1D domains, making use of the $\gamma=r$ propagator derived in Eq. (2), one can straightforwardly derive the relations for reflecting boundaries:

$$
\tilde{R}_{n}^{(r)}(z)=1-\frac{\sqrt{z^{2}(1-2 q)-2 z(1-q)+1} \sinh [N \zeta(z)]}{2 \cosh \left[\left(N-n+\frac{1}{2}\right) \zeta(z)\right] \cosh \left[\left(n-\frac{1}{2}\right) \zeta(z)\right]}
$$

and

$$
\tilde{F}_{n_{0}}^{(r)}(n, z)=\frac{\cosh \left[\left(N-n_{>}+\frac{1}{2}\right) \zeta(z)\right] \cosh \left[\left(n_{<}-\frac{1}{2}\right) \zeta(z)\right]}{\cosh \left[\left(N-n+\frac{1}{2}\right) \zeta(z)\right] \cosh \left[\left(n-\frac{1}{2}\right) \zeta(z)\right]} .
$$

As expected, Eqs. (8) and (9) depend explicitly on $n$ and $n_{0}$. This dependence is not the case for the periodic case for which translational invariance makes the return probability

$$
\tilde{R}^{(p)}(z)=1-\frac{\sqrt{z^{2}(1-2 q)-2 z(1-q)+1} \sinh [N \zeta(z)]}{\cosh [N \zeta(z)]+1},
$$

independent of $n$ and the first-passage probability

$$
\tilde{F}_{n_{0}}^{(p)}(n, z)=\frac{\cosh \left[\left(\frac{N}{2}-\left|n-n_{0}\right|\right) \zeta(z)\right]}{\cosh \left[\frac{N}{2} \zeta(z)\right]},
$$

dependent only on the difference $n-n_{0}$. The analytic time dependence of Eqs. (8) and (10) is reported, respectively, in Eqs. (S3) and (S5) of Ref. [75], while the one for Eqs. (9) and (11) can be found, respectively, in Eqs. (C2) and (C4).

The first-passage probability to either of the two boundaries, $\mathcal{E}_{n_{0} \rightarrow(1 ; N)}(t)$, one at $n=1$ and one at $n=N$, is

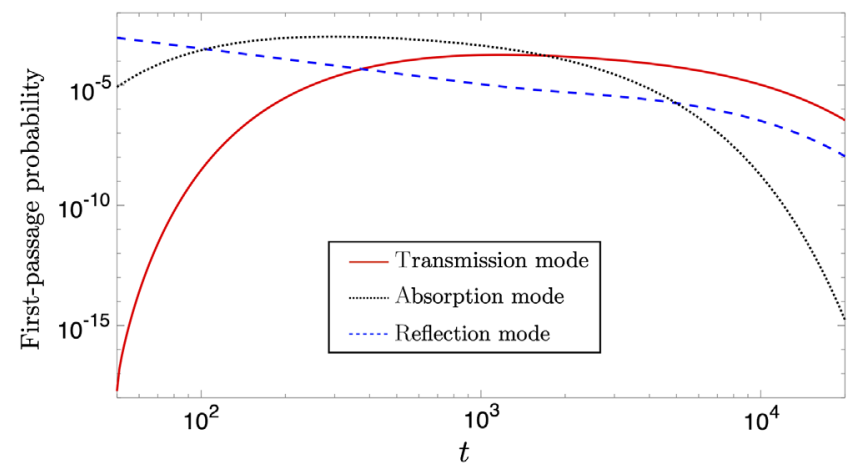

FIG. 2. Time dependence of the first-passage probability in a $1 \mathrm{D}$ domain of size $N=51$ with $q=0.7$. The curve in the absorption mode is the plot of $\mathcal{E}_{n_{0} \rightarrow(1 ; N)}(t)$ in Eq. (C5) with $n_{0}=25$. The reflection and transmission modes display $F_{n_{0}}^{(r)}(N, t)$ from Eq. (C2) with, respectively, $n_{0}=5$ and $n_{0}=50$. The reflection mode exhibits a power-law dependence in time, whereas an exponential dependence is present for the transmission and absorption modes.

related to the survival probability, $\mathcal{S}_{n_{0}}(t)$, via $\mathcal{E}_{n_{0} \rightarrow(1 ; N)}(t)=$ $\mathcal{S}_{n_{0}}(t-1)-\mathcal{S}_{n_{0}}(t)$ with $\mathcal{S}_{n_{0}}(t)=\sum_{n=1}^{N} P_{n_{0}}^{(a)}(n, t) \quad$ [see explicit expression for $\mathcal{E}_{n_{0} \rightarrow(1 ; N)}(t)$ in Eq. (C5)]. In transport calculations [43], one refers to this case as the absorption mode, to distinguish it from the reflection mode and transmission mode, which occur when both an absorption and a reflecting lattice site are present. If the initial condition is near the absorbing (reflecting) boundary, one refers to it as the reflection (transmission) mode. The first-passage dynamics is very different in the two modes. In the reflection mode, the problem is similar to the firstpassage probability in an unbounded domain, and the time dependence has a very slow, power-law-like dynamics, whereas at later times, once the reflecting boundary has been reached, the dynamics is exponential. In the transmission mode, with only the length scale of the size of the domain affecting the dynamics, an exponential dependence is instead already present at short times. In Fig. 2, I show these different dynamics by plotting, on a log-log plot, the time dependence of the first-passage probability in the absorption, transmission, and reflection modes.

Another quantity directly related to the propagator generating function is the number of distinct sites visited. While finding its probability is a formidable challenge, the mean is readily accessible and given by [19]

$$
\widetilde{\mathcal{M}}_{\vec{n}_{0}}(z)=\frac{1}{(1-z)^{2} \tilde{P}_{\vec{n}_{0}}(\vec{n}, z)},
$$

written explicitly in vectorial notation since it will be used later in Sec. VB when dealing with LRW in higher dimensions. Through a residue calculation, the 1D case can also be found analytically [see Eqs. (D1) and (D3), respectively, for reflecting and periodic boundaries]. 


\section{MEAN FIRST-PASSAGE TIME IN ONE DIMENSION}

In various instances, the estimation of the mean of the first-passage probability, that is, the MFPT, provides a valuable estimate with which to interpret empirical findings, e.g., agent reactivity in chemical reactions [76] and animal foraging efficiency [30]. Analytic knowledge of the generating function of the first-passage probability provides a convenient way to compute the MFPT via

$$
T_{\vec{n}_{0} \rightarrow \vec{n}}=\left.\frac{\mathrm{d} \tilde{F}_{\vec{n}_{0}}(\vec{n}, z)}{\mathrm{d} z}\right|_{z=1},
$$

a well-known relation, which is also valid for arbitrary dimensions.

In 1D, the MFPT for the reflecting and periodic cases can be derived by taking the generating function of the propagator in Eq. (5) and, subsequently, exploiting the identities (E2) and (E4), resulting in the compact expression

$T_{n_{0} \rightarrow n}^{(r)}=\frac{1}{q}\left[N\left|n-n_{0}\right|+\left(n-n_{0}\right)\left(n+n_{0}-1-N\right)\right]$,

for the reflecting domain, and

$$
T_{n_{0} \rightarrow n}^{(p)}=\frac{1}{q}\left(N-\left|n-n_{0}\right|\right)\left|n-n_{0}\right|,
$$

for the periodic domain. Comparison of the two expressions shows that there are values for $n$ for which $T_{n_{0} \rightarrow n}^{(p)}>T_{n_{0} \rightarrow n}^{(r)}$. It is easier to determine the range of such values by considering separately $N$ odd or even. When $N$ is odd, and $n_{0}>(N+1) / 2, T_{n_{0} \rightarrow n}^{(p)}>T_{n_{0} \rightarrow n}^{(r)}$ whenever $(N+1) / 2<n<n_{0}$, while $T_{n_{0} \rightarrow n}^{(p)}=T_{n_{0} \rightarrow n}^{(r)}$ when $n_{0}=(N+3) / 2$. Similarly, when $n_{0}<(N+1) / 2$, $T_{n_{0} \rightarrow n}^{(p)}>T_{n_{0} \rightarrow n}^{(r)}$ whenever $n_{0}<n<(N+1) / 2$ and $T_{n_{0} \rightarrow n}^{(p)}=$ $T_{n_{0} \rightarrow n}^{(r)}$ when $n_{0}=(N-1) / 2$. In all other cases, $T_{n_{0} \rightarrow n}^{(p)}<$ $T_{n_{0} \rightarrow n}^{(r)}$. This result is intuitively understandable as the range of values found corresponds to, with $n_{0}>(N+1) / 2$, the case when the target position $n$ is to the right of the halfway point in the domain and $n_{0}$ is in between the target site $n$ and lattice site $n=N$. The presence of the boundary at $n=N$ increases the chance of reaching $n$ when compared to the periodic case for which trajectories may also reach $n$ by taking the longer route by moving mostly to the right and then reaching $n$ from the left. The other case, that is, when $n_{0}<(N+1) / 2$, corresponds to when the target position $n$ is to the left of the halfway point in the domain and $n_{0}$ is in between the target site $n$ and lattice site $n=1$.
When $N$ is even, one has a slight modification: $T_{n_{0} \rightarrow n}^{(p)}$ cannot be equal to $T_{n_{0} \rightarrow n}^{(r)}$, and with $n_{0}>\lceil(N+1) / 2\rceil(\lceil x\rceil$, called the "ceiling" of $x$, represents the least integer greater than or equal to $x$ ), one has $T_{n_{0} \rightarrow n}^{(p)}>T_{n_{0} \rightarrow n}^{(r)}$ when $\lceil(N+1) / 2\rceil \leq n<n_{0}$; however, with $n_{0}>\lfloor(N+1) / 2\rfloor$ $(\lfloor x\rfloor$, called the "floor" of $x$, represents the greatest integer less than or equal to $x$ ), one has $T_{n_{0} \rightarrow n}^{(p)}>T_{n_{0} \rightarrow n}^{(r)}$ when $n_{0}<n \leq\lfloor(N+1) / 2\rfloor$. For all other cases, $T_{n_{0} \rightarrow n}^{(p)}<T_{n_{0} \rightarrow n}^{(r)}$.

From the first-passage probability $\mathcal{E}_{n_{0} \rightarrow(1 ; N)}(t)$, it is straightforward to determine the mean time to either of the two boundaries, $T_{n_{0} \rightarrow(1 ; N)}=\sum_{t=0}^{\infty} t \mathcal{E}_{n_{0} \rightarrow(1 ; N)}(t)$, the so-called mean exit time. The resulting expression is the counterpart of the well-known continuous space-time result (see, e.g., Ref. [77]) and is given by

$$
T_{n_{0} \rightarrow(1 ; N)}=\frac{1}{q}\left(N-n_{0}\right)\left(n_{0}-1\right) .
$$

\section{PROPAGATORS IN HIGHER DIMENSIONS}

\section{A. Two-dimensional propagator with reflecting boundaries}

I start with $2 \mathrm{~d}$, in particular, analyzing the case of a domain of size $N_{1} \times N_{2}$ with four reflecting boundaries. To proceed, one needs to first consider an analogous problem, that of a LRW confined along one direction, characterized by coordinates $n_{1}\left(1 \leq n_{1} \leq N_{1}\right)$, and unbounded along the other, characterized by coordinates $n_{2}$. Away from the boundaries, the walker along each coordinate has a chance to move to a neighboring site with probability $q_{i} / 4$, with $i=1$ and 2 , and a chance to stay with probability $1-\left(q_{1}+q_{2}\right) / 2$. The discrete master equation governing the 2D dynamics of the semibounded probability $\mathcal{P}\left(n_{1}, n_{2}, t\right)$ in the bulk ( $\mathcal{P}$ has been chosen to distinguish it from the fully bounded propagator $P$ ), that is, when away from any of the boundaries, is given by

$$
\begin{aligned}
\mathcal{P} & \left(n_{1}, n_{2}, t+1\right) \\
= & \left(1-\frac{q_{1}+q_{2}}{2}\right) \mathcal{P}\left(n_{1}, n_{2}, t\right) \\
& +\frac{q_{1}}{4}\left[\mathcal{P}\left(n_{1}-1, n_{2}, t\right)+\mathcal{P}\left(n_{1}+1, n_{2}, t\right)\right] \\
& +\frac{q_{2}}{4}\left[\mathcal{P}\left(n_{1}, n_{2}-1, t\right)+\mathcal{P}\left(n_{1}, n_{2}+1, t\right)\right],
\end{aligned}
$$

and for any site with coordinates $\left(1, n_{2}\right)$ and $\left(N_{1}, n_{2}\right)$, the dynamics of the probability satisfies the following two equations, respectively: 


$$
\begin{aligned}
\mathcal{P}\left(1, n_{2}, t+1\right) & =\left(\frac{1}{2}-\frac{q_{1}}{4}+\frac{1-q_{2}}{2}\right) \mathcal{P}\left(1, n_{2}, t\right)+\frac{q_{1}}{4} \mathcal{P}\left(2, n_{2}, t\right)+\frac{q_{2}}{4}\left[\mathcal{P}\left(1, n_{2}-1, t\right)+\mathcal{P}\left(1, n_{2}+1, t\right)\right], \\
\mathcal{P}\left(N, n_{2}, t+1\right) & =\left(\frac{1}{2}-\frac{q_{1}}{4}+\frac{1-q_{2}}{2}\right) \mathcal{P}\left(N, n_{2}, t\right)+\frac{q_{1}}{4} \mathcal{P}\left(N-1, n_{2}, t\right)+\frac{q_{2}}{4}\left[\mathcal{P}\left(N, n_{2}-1, t\right)+\mathcal{P}\left(N, n_{2}+1, t\right)\right] .
\end{aligned}
$$

I first seek the solution of the semibounded problem defined in Eqs. (17) and (18) with initial conditions $\mathcal{P}\left(n_{1}, n_{2}, 0\right)=\delta_{n_{1}, n_{01}} \delta_{n_{2}, n_{02}}$.

With the movement along the vertical axis being unrestricted, one can Fourier transform Eqs. (17) and (18) and write an effective 1D discrete master equation analogous to Eq. (7) of the form

$$
\hat{\mathcal{P}}\left(n_{1}, \kappa_{2}, t+1\right)=\sum_{\ell=1}^{N_{1}} \mathbb{B}_{n_{1} \ell} \hat{\mathcal{P}}\left(\ell, \kappa_{2}, t\right),
$$

where $\hat{\mathcal{P}}\left(n_{1}, \kappa_{2}, t\right)=\sum_{n_{2}=-\infty}^{+\infty} e^{-i \kappa_{2} n_{2}} \mathcal{P}\left(n_{1}, n_{2}, t\right)$. The matrix $\mathbb{B}$ is tridiagonal with elements of the upper and lower diagonals equal to $q_{1} / 4$, and with diagonal elements $\mathbb{B}_{\ell \ell}=1-q_{1} / 2-q_{2}\left[1-\cos \left(\kappa_{2}\right)\right] / 2$ when $\ell \neq 1, N_{1}$ and $\mathbb{B}_{11}=\mathbb{B}_{N_{1} N_{1}}=1-q_{1} / 4-q_{2}\left[1-\cos \left(\kappa_{2}\right)\right] / 2$. As matrix $\mathbb{B}$ is a Toeplitz matrix with perturbed corners, the exact solution is of the same form of Eq. (5), namely,

$$
\hat{\mathcal{P}}_{\vec{n}_{0}}\left(n_{1}, \kappa_{2}, t\right)=\sum_{k_{1}=0}^{N_{1}-1} \frac{\alpha_{k_{1}}}{N_{1}} \cos \left[\left(n_{1}-\frac{1}{2}\right) \frac{\pi k_{1}}{N_{1}}\right] \cos \left[\left(n_{01}-\frac{1}{2}\right) \frac{\pi k_{1}}{N_{1}}\right]\left\{1-\frac{q_{1}}{2}+\frac{q_{1}}{2} \cos \left(\frac{\pi k_{1}}{N_{1}}\right)-\frac{q_{2}}{2}+\frac{q_{2}}{2} \cos \left(\kappa_{2}\right)\right\}^{t} e^{-i \kappa_{2} n_{02}},
$$

where the symbol $\vec{n}_{0}$ stands for $\left(n_{01}, n_{02}\right)$. The next step requires the generating function of Eq. (20) and, subsequently, its inverse Fourier transform. Then, employing the method of images to the resulting expression for reflecting boundaries at $n_{2}=1$ and $n_{2}=N_{2}$ provides the generating function of the exact solution in the fully bounded 2D domain. And finally, through an inverse $z$ transformation, one obtains the exact spatiotemporal dependence (see further details in Ref. [75]),

$$
\begin{aligned}
P_{\vec{n}_{0}}\left(n_{1}, n_{2}, t\right)= & \frac{1}{N_{1} N_{2}} \sum_{k_{1}=0}^{N_{1}-1} \sum_{k_{2}=0}^{N_{2}-1} \alpha_{k_{1}} \alpha_{k_{2}} \cos \left[\left(n_{1}-\frac{1}{2}\right) \frac{\pi k_{1}}{N_{1}}\right] \\
& \times \cos \left[\left(n_{01}-\frac{1}{2}\right) \frac{\pi k_{1}}{N_{1}}\right] \cos \left[\left(n_{2}-\frac{1}{2}\right) \frac{\pi k_{2}}{N_{2}}\right] \cos \left[\left(n_{02}-\frac{1}{2}\right) \frac{\pi k_{2}}{N_{2}}\right] \\
& \times\left[1-\frac{q_{1}+q_{2}}{2}+\frac{q_{1}}{2} \cos \left(\frac{\pi k_{1}}{N_{1}}\right)+\frac{q_{2}}{2} \cos \left(\frac{\pi k_{2}}{N_{2}}\right)\right]^{t}
\end{aligned}
$$

with $\alpha_{k_{i}}=1$ when $k_{i}=0$ and 2 otherwise.

In Fig. 3, I plot Eq. (21) and the numerical (iterative) solution of the equation

$$
P\left(n_{1}, n_{2}, t+1\right)=\sum_{\ell=1}^{N_{1}} \sum_{r=1}^{N_{2}} \mathbb{A}_{n_{1} \ell, n_{2} r} P(\ell, r, t),
$$

where $\mathbb{A}_{n \ell, m r}$ is a fourth-order tensor that accounts for the bulk dynamics as well as the dynamics along the four boundaries. With the walker always moving $\left(q_{1}=q_{2}=1\right)$, at short enough times, parity dependencies appear in the shape of $P\left(n_{1}, n_{2}, t\right)$, for which, at odd (even) times, only locations that are an odd (even) number of steps away from the initial position have nonzero probability. In this case, it is evident that all $\left(n_{1}, n_{2}\right)$ elements for which $n_{1}+n_{2}-$ $n_{01}-n_{02}$ is even have $P\left(n_{1}, n_{2}, 5\right)=0$. As the walker is equally likely to be found anywhere at long times$\left(N_{1} N_{2}\right)^{-1}$ is the long time limit of Eq. (21) - the walker gradually loses this parity-dominated dynamics after reaching any boundary point where it always has a chance to stay.

\section{B. Propagator in arbitrary dimensions and arbitrary boundary conditions}

The procedure to derive the $2 \mathrm{D}$ analytic propagator for the case of four reflecting boundaries can be followed to construct the solution in arbitrary dimensions for a bounded domain of size $\prod_{i} N_{i}$ with any combination of boundary conditions along each direction $i$ and any parameter $0<q_{i} \leq 1$, with $q_{i} /(2 d)$ representing the jump probability to either of the nearest-neighbor sites along direction $i$. It is 


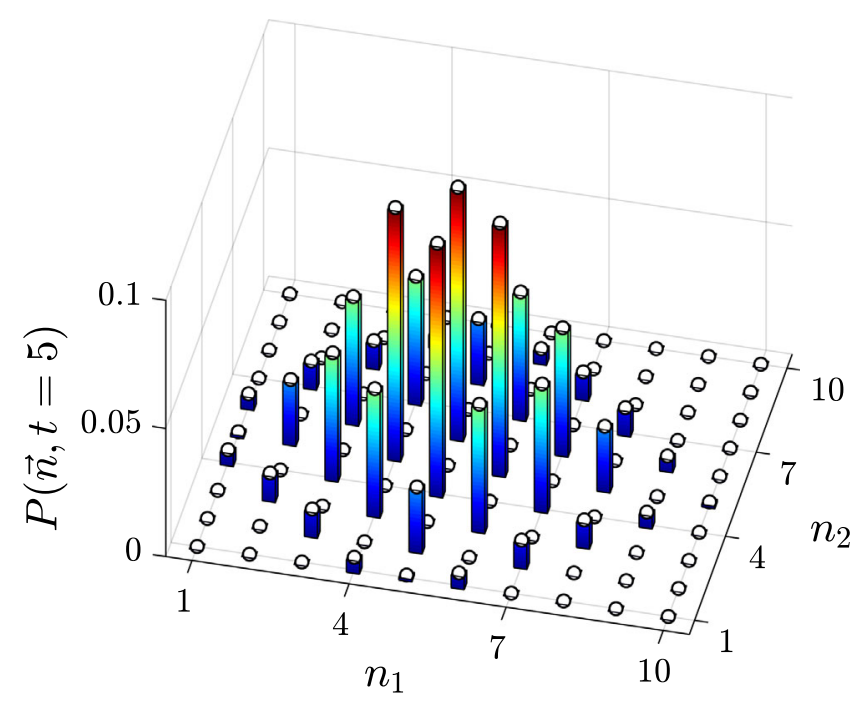

FIG. 3. Two-dimensional propagator in a lattice with reflecting boundaries and size $N=M=10$ at time $t=5$ starting from the initial condition $P\left(n_{1}, n_{2}, 0\right)=\delta_{n_{1}, 5} \delta_{n_{2}, 5}$. The open circles are obtained from the analytic expression in Eq. (21) with $q_{1}=q_{2}=1$, while the colored bars are obtained by iteratively solving Eq. (22).

an eight-step hierarchical construction based on the knowledge of the analytic solution of the associated problem in $d-1$ dimensions. For ease of understanding, a visual representation of this hierarchical construction is drawn in Fig. S1 in Ref. [75], where some additional details of the derivation are also reported. The first step consists of writing a master equation for a LRW in $d$ dimensions, whose dynamics represent a walker moving in unbounded space along the $d$ th dimension while confined along the remaining $d-1$ dimensions. The dynamics governing the walker movement when at a boundary site along any of the $d-1$ bounded dimensions are chosen according to the specified boundary conditions. In the second step, after a Fourier transformation along the $d$ th dimension is performed, one is left with a master equation for a walker in an effective $d$-1-dimensional space. The analytic solution of this master equation can be found by realizing that the only difference from the solution of the fully bounded problem in $d-1$ dimensions is that the probability of staying contains the cosine of the Fourier coefficients along the unbounded $d$ th direction in the bulk and the appropriate correction when at any of the boundary sites. In the third step, one can thus write the time-dependent propagator for the $d-1$ bounded dimensions, which contains as parameters Fourier coefficients associated with the $d$ th dimension. The fourth and fifth steps involve, respectively, writing the generating function of the propagator and then its inverse Fourier transform. With the resulting generating function of the propagator now bounded along $d-1$ dimensions and unbounded along the $d$ th dimension, the method of images, for the boundary conditions specified by the problem, along the $d$ th dimension is employed in the sixth step to obtain the generating function for the fully bounded propagator. In the seventh step, the propagator generating function is rewritten in terms of Chebyshev polynomials, and the appropriate identities presented in Appendix E are used to obtain the propagator generating function in terms of $d$ finite nested sums. The eighth (and final) step is an inverse $z$ transform to obtain the following spatiotemporal dependence of the propagator in $d$ dimensions:

$$
\begin{aligned}
P_{\vec{n}_{0}}\left(n_{1}, \ldots, n_{d}, t\right)= & \sum_{k_{1}=w_{1}^{\left(\gamma_{1}\right)}}^{\omega_{1}^{\left(\gamma_{1}\right)}} \ldots \sum_{k_{d}=w_{d}^{\left(\gamma_{d}\right)}}^{\omega_{d}^{\left(\gamma_{d}\right)}}\left[\prod_{i=1}^{d} g_{k_{i}}^{\left(\gamma_{i}\right)}\left(n_{i}, n_{0 i}\right)\right] \\
& \times\left[\frac{s_{k_{1}}^{\left(\gamma_{1}\right)}\left(q_{1}\right)}{d}+\cdots+\frac{s_{k_{d}}^{\left(\gamma_{d}\right)}\left(q_{d}\right)}{d}\right]^{t} \cdot
\end{aligned}
$$

Recall that the function $s_{k}^{(\gamma)}(q)$ and $g_{k}^{(\gamma)}\left(n, n_{0}\right)$ are defined, respectively, in Eqs. (4) and (6), that the lower summati on limit $w^{(\gamma)}=0$ when $\gamma=r$ or $p, w^{(\gamma)}=1$ when $\gamma=a$ or $m$, and that the upper summation limit $\omega^{(\gamma)}=N-1$ when $\gamma=r, p$, or $m$ and $\omega^{(a)}=N-2$.

The structure of the general propagator in Eq. (23) is relatively simple. It is made up of a nested finite series whereby the spatial components (eigenbases) appropriate for the boundary conditions along each direction are multiplied, while the temporal dependence for each direction is summed first and then evaluated to the power $t$.

The advantage of the analytic expression for $P_{\vec{n}_{0}}\left(n_{1}, \ldots\right.$, $\left.n_{d}, t\right)$ is that it bypasses the construction of the appropriate multidimensional array that represents the jump transition probability. It thus avoids the need to iteratively solve the discrete master equation, whose computational cost would scale as $\left[(2 d+1) \prod_{i=1}^{d} N_{i}\right] t$, where $2 d+1$ stems from the number of allowed nearest-neighbor jump probability for a Pólya's walk in a space of dimensions $d$. This linear time cost should be compared to $\left(\prod_{i=1}^{d} N_{i}\right) \log \left(\prod_{i=1}^{d} N_{i}\right)$ when computing the nested sums in Eq. (23) at any given time $t$, e.g., through the use of fast Fourier transforms. In other words, by using the analytics, there is a gain in computation from being linearly dependent on time to be independent from it.

The convenience in employing the exact propagators is particularly evident when analyzing the dynamics for derived quantities that have been so far accessible only via stochastic simulations. An example is the time dependence of the average number of distinct visited sites, $\mathcal{M}_{\vec{n}_{0}}(t)$, whose generating function has been defined in Eq. (12). In a box of size $N^{d}$, even with the choice $N=10$, the computational cost to generate stochastic simulation outputs is prohibitive as $d$ grows, while the numerical $z$ inversion of the analytic expression allows one to plot in Fig. 4 the resulting dynamics straightforwardly. From the 


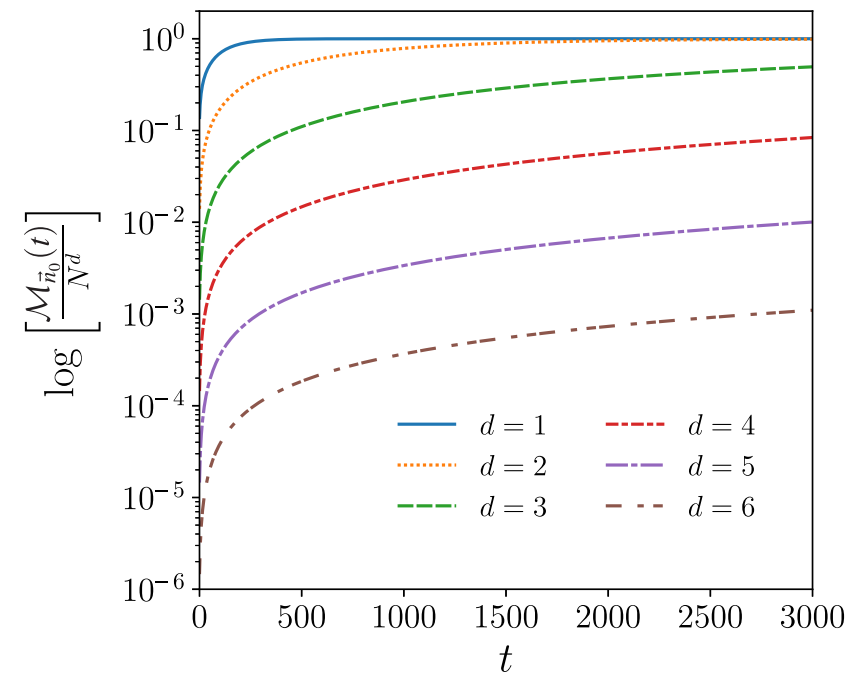

FIG. 4. Time dependence of the average number of distinct visited sites in a box (with reflective boundary conditions along each side) of size $N^{d}$. The 1D case is plotted by using Eq. (D1), while all the remaining ones are obtained through a numerical $z$ inversion of Eq. (12). The starting location, for $i=1, \ldots, d$, is at $n_{0 i}=1$, and $q_{i}=2 d /(2 d+1)$ so that in any dimension the transition probabilities at each time step to move to either of the adjacent sites, $q_{i} /(2 d)$, or to stay, $1-\sum_{i=1}^{d} q_{i} /(d)$, are all identical, and equal to $1 /(2 d+1)$, when away from the boundaries.

figure, it is noticeable that the cases for $d=1$ and $d=2$ are qualitatively different from the remaining ones. This difference is due to the recurrent or transient nature of the associated Pólya's walks in unbounded space, which are recurrent in $1 \mathrm{D}$ and $2 \mathrm{D}$, and transient in $d \geq 3[15,16]$.

\section{Propagators in the continuous limit}

Known results in the continuous time limit can be recovered with standard procedures (see, e.g., Ref. [24]) by considering the propagator $\mathcal{C}_{\vec{n}_{0}}\left(n_{1}, n_{2}, \ldots, n_{d}, \tau\right)=$ $\sum_{s=0}^{\infty} W(s, \tau) P_{\vec{n}_{0}}\left(n_{1}, n_{2}, \ldots, n_{d}, s\right)$, where $W(s, \tau)$ is the probability for $s$ jump events to occur in (continuous) time $\tau$. With $\psi(\tau)$, the waiting time probability distribution for a jump event to occur at time $\tau$, one can construct $\bar{W}(s, \epsilon)=$ $\{[1-\bar{\psi}(\epsilon)] / \epsilon\} \bar{\psi}^{s}(\epsilon)$, where $\bar{f}(\epsilon)=\int_{0}^{\infty} d t e^{-\epsilon t} f(t)$ is the Laplace transform of $f(t)$, and carry through the infinite summation to obtain

$$
\begin{aligned}
& \overline{\mathcal{C}}_{\vec{n}_{0}}\left(n_{1}, n_{2}, \ldots, n_{d}, \epsilon\right) \\
& =\sum_{k_{1}=w_{1}^{\left(\gamma_{1}\right)}}^{\omega_{1}^{\left(\gamma_{1}\right)}} \ldots \sum_{k_{d}=w_{d}^{\left(\gamma_{d}\right)}}^{\omega_{d}^{\left(\gamma_{d}\right)}} \frac{[1-\bar{\psi}(\epsilon)] \prod_{i=1}^{d} g_{k_{i}}^{\left(\gamma_{i}\right)}\left(n_{i}, n_{0 i}\right)}{\left.\epsilon 1-\bar{\psi}(\epsilon)\left[\frac{s_{k_{1}}^{\left(\gamma_{1}\right)}\left(q_{1}\right)}{d}+\cdots+\frac{s_{k_{d}}^{\left(\gamma_{d}\right)}\left(q_{d}\right)}{d}\right]\right\}} .
\end{aligned}
$$

With the choice $\psi(\tau)=2 d R e^{-2 d R \tau}$, with $R$ a rate [i.e., $\bar{\psi}(\epsilon)=2 d R /(\epsilon+2 d R)$ ], and subsequent Laplace inversion, one recovers the factorized form characteristic of the discrete space and continuous time limit,

$$
\mathcal{C}_{\vec{n}_{0}}\left(n_{1}, n_{2}, \ldots, n_{d}, \tau\right)=\prod_{i=1}^{d} C_{n_{0 i}}^{\left(\gamma_{i}\right)}\left(n_{i}, \tau\right)
$$

with

$C_{n_{0 i}}^{\left(\gamma_{i}\right)}\left(n_{i}, \tau\right)=\sum_{k_{i}=w_{i}^{\left(\gamma_{i}\right)}}^{\omega_{i}^{\left(\gamma_{i}\right)}} g_{k}^{\left(\gamma_{i}\right)}\left(n_{i}, n_{0 i}\right) e^{-2 R q_{i} \tau\left[1-\cos \left(\pi \mathcal{N}_{k_{i}}^{\left(\gamma_{i}\right)}\right)\right]}$,

where the various symbols are the same as in Eq. (23). The analytic form of $C_{n_{0 i}}^{(\gamma)}\left(n_{i}, \tau\right)$ can also be found in multiple sources; see, e.g., Refs. [71,78,79].

From Eq. (25), for the continuous spatial limit, one considers a lattice spacing of size $b$, with $b \rightarrow 0, N_{i}, n, n_{0}$, $R \rightarrow+\infty$, such that $x=n b, x_{0}=n_{0} b, L_{i}=N_{i} b$, with $L_{i}$ the domain size $\left(0 \leq x_{i}, x_{0 i} \leq L_{i}\right)$, and $q_{i} R b^{2} \rightarrow D_{i}$, with $D_{i}$ the diffusion constant. With the above limits, Eq. (26) transforms into

$$
C_{x_{0 i}}^{(\gamma)}\left(x_{i}, \tau\right)=\sum_{k=\bar{k}_{\gamma}}^{+\infty} h_{k}^{(i, \gamma)}\left(x_{i}, x_{0 i}\right) e^{-\left(D_{i} \pi^{2} k_{\gamma}^{2} / L_{i}^{2}\right) \tau},
$$

where $\quad h_{k}^{(i, r)}\left(x, x_{0}\right)=\alpha_{k} \cos \left(\pi k x / L_{i}\right) \cos \left(\pi k x_{0} / L_{i}\right) / L_{i}$, $h_{k}^{(i, p)}\left(x, x_{0}\right)=\cos \left[2 \pi k\left(x-x_{0}\right) / L_{i}\right] / L_{i}, \quad h_{k}^{(i, a)}\left(x, x_{0}\right)=$ $2 \sin \left(\pi k x / L_{i}\right) \sin \left(\pi k x_{0} / L_{i}\right) / L_{i}, \quad$ and $\quad h_{k}^{(i, m)}\left(x, x_{0}\right)=$ $2 \cos \left[\pi(k-1 / 2) x / L_{i}\right) \cos \left[\pi(k-1 / 2) x_{0} / L_{i}\right] / L_{i}$, and with $\bar{k}_{r, p}=0, \bar{k}_{a, m}=1, k_{r, a}=k, k_{p}=2 k, k_{m}=(2 k-1) / 2$. These functional dependencies match the space-time continuous propagators found in multiple references (see, e.g., Refs. [80-82]).

In Sec. II, it was mentioned that the parameter controlling the probability to move to nearest-neighboring sites is only a rescaling of the jump rate in continuous time or the diffusion constant in the space-time continuous limit. From Eq. (26), one can clearly see that $q_{i}$ is a rescaling of the jump rate $R$ between lattice sites along each direction, or a rescaling of the diffusion constant along each direction, when inspecting Eq. (27).

\section{DYNAMICS IN THE PRESENCE OF A DEFECTIVE SITE}

Besides boundary sites, in many finite systems there exist other special spatial locations, often called defects, that affect the movement steps of a random walker. These defects may either change the probability of a walker to move to a neighboring site $[83,84]$, or they may be partially absorbing traps [21,22]. The absorbing case is particularly important and has been the subject of past [85-87] and renewed interest $[88,89]$. In the presence of partially 

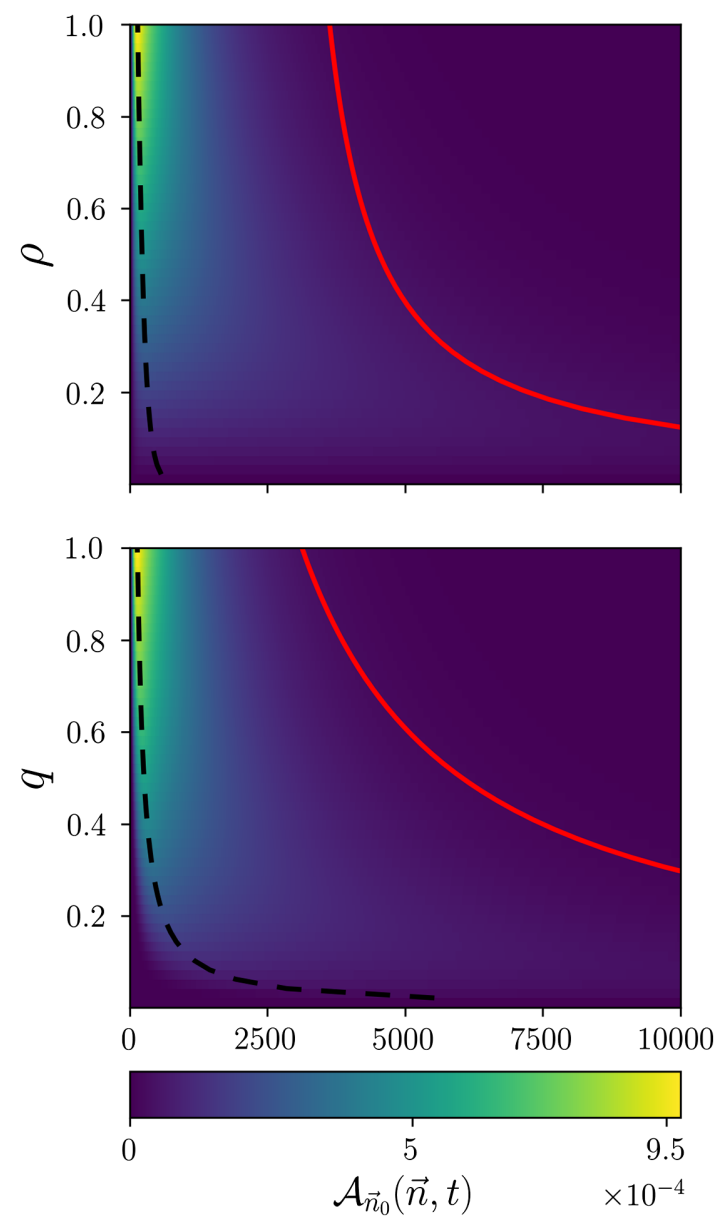

FIG. 5. Plot of the absorption probability $\mathcal{A}_{\vec{n}_{0}}(\vec{n}, t)$ in a $2 \mathrm{D}$ periodic domain of size $30 \times 30$ as a function of time obtained from a numerical $z$ inversion of Eq. (28) with the walker having $q_{1}=q_{2}=q$ in $\tilde{P}_{\vec{n}_{0}}(\vec{n}, z)$. The partially absorbing target is located at $\vec{n}=(21,9)$, and the initial condition is at site $\vec{n}_{0}=(4,18)$. The top panel is drawn for absorption values in the range $0.001 \leq \rho \leq 1$ and $q=0.8$, while the bottom panel is drawn for $0.001 \leq q \leq 1$ and $\rho=0.8$. The solid line is the mean of the distributions, while the dashed line represents the mode.

absorbing traps, two temporal scales affect the LRW dynamics: the time it takes for a walker to reach either of the traps and the reaction time, that is, the time to be absorbed at the trap site. The former is controlled by the dynamics of the first-passage probability to reach either of the traps, while the latter is controlled by the probability $\rho$ of being absorbed when at one of the traps. While there exist two limiting cases [90,91] — the reaction-limited case when $\rho \ll 1$, and the motion limited case, also called geometrycontrolled limit, when $q_{i} \ll 1$-in general, there is a rich temporal dependence as a function of $\rho, q_{i}$, the domain size and the initial location relative to the trap [92]. To describe the dynamics in the general case, the full time dependence of the absorption probability becomes necessary.

For the simple case of one trap, one can apply the wellknown defect technique $[93,94]$ and write the generating function of the absorption probability at site $\vec{n}$ starting from site $\vec{n}_{0}$ as

$$
\tilde{\mathcal{A}}_{\vec{n}_{0}}(\vec{n}, z)=\frac{\tilde{P}_{\vec{n}_{0}}(\vec{n}, z)}{\frac{1}{\rho}-1+\tilde{P}_{\vec{n}}(\vec{n}, z)} .
$$

In Fig. 5, I show the time dependence for the case of a single trap in a 2D domain of size $N \times N$ with periodic boundaries. The difference between the mode of the absorption probability and the mean absorption time,

$$
A_{\vec{n}_{0} \rightarrow \vec{n}}=T_{\vec{n}_{0} \rightarrow \vec{n}}+\left(\frac{1}{\rho}-1\right) N^{d}
$$

is strikingly visible and points to different regimes of temporal dependence as observed for Brownian walks $[92,95,96]$.

\section{MEAN FIRST-PASSAGE TIME TO A SINGLE TARGET AND PSEUDO-GREEN FUNCTIONS IN ARBITRARY DIMENSIONS}

Knowledge of the propagator in arbitrary dimensions gives one the ability to analytically determine the MFPT to a single target for periodic and reflecting domains for any $d$ as a finite series of a nested sum. Here, I present the MFPT when boundaries are all reflecting or all periodic, but it is straightforward to construct the case when there is a mixture of reflecting and periodic boundaries along different directions.

As mentioned earlier in Sec. IV, the MFPT is obtained using Eq. (13) and can be written in a compact way by first defining the $d$-dimensional set of vectors $\vec{I}=I_{1}, \ldots, I_{d}$, with $I_{i}=\{0,1\}$, which has $2^{d}$ possible elements, and considering the function

$$
\begin{aligned}
K(\vec{k})= & \left\{\cos ^{2}\left[\left(n_{1}-\frac{1}{2}\right) \frac{\pi k_{1}}{N_{1}}\right] \ldots \cos ^{2}\left[\left(n_{d}-\frac{1}{2}\right) \frac{\pi k_{d}}{N_{d}}\right]\right. \\
& -\cos \left[\left(n_{1}-\frac{1}{2}\right) \frac{\pi k_{1}}{N_{1}}\right] \cos \left[\left(n_{01}-\frac{1}{2}\right) \frac{\pi k_{1}}{N_{1}}\right] \ldots \\
& \left.\ldots \times \cos \left[\left(n_{d}-\frac{1}{2}\right) \frac{\pi k_{d}}{N_{d}}\right] \cos \left[\left(n_{0 d}-\frac{1}{2}\right) \frac{\pi k_{d}}{N_{d}}\right]\right\} \\
& \times\left\{\sum_{i=1}^{d} q_{i}\left[1-\cos \left(\frac{\pi k_{i}}{N_{i}}\right)\right]\right\}^{-1} .
\end{aligned}
$$

The MFPT to a single target in a $d$-dimensional box (reflecting boundaries) is given by

$$
T_{\vec{n}_{0} \rightarrow \vec{n}}=d \sum_{i=1}^{d} 2^{i} \sum_{\vec{k}}^{\vec{N}} \sum_{\vec{I} \in M_{i}} K(\vec{I} \cdot \vec{k}),
$$

where $\sum_{\vec{k}}^{\vec{N}}$ represents $d$ summations for $k_{i}=1, \ldots, N_{i}$, with $i=1, \ldots, d$, and $M_{i}=\left\{\vec{x} \in \vec{I}: \sum_{j=1}^{d} x_{j}=i\right\}$; that is, $M_{i}$ represents the subset of all combinations of the vectors 
in $\vec{I}$ that have $d-i$ elements equal to zero. Application of Eq. (31) for a 4D lattice with reflecting boundaries is presented later in Sec. VIII B where an analysis of the MFPT to either of a set of three targets is carried out.

Equations (30) and (31) allow me to make contact with the pseudo-Green function formalism developed to analytically calculate the MFPT and a wealth of other quantities in 1D, 2D, and 3D for LRWs that move at each time step via [45,97-99]

$$
T_{\vec{n}_{0} \rightarrow \vec{n}}=\left(\prod_{i=1}^{d} N_{i}\right)\left[H(\vec{n} \mid \vec{n})-H\left(\vec{n} \mid \overrightarrow{n_{0}}\right)\right] .
$$

By using

$$
\begin{aligned}
\mathcal{H}^{(r)}(\vec{k})= & \left\{\cos \left[\left(n_{1}-\frac{1}{2}\right) \frac{\pi k_{1}}{N_{1}}\right] \cos \left[\left(n_{01}-\frac{1}{2}\right) \frac{\pi k_{1}}{N_{1}}\right] \ldots\right. \\
& \left.\times \cos \left[\left(n_{d}-\frac{1}{2}\right) \frac{\pi k_{d}}{N_{d}}\right] \cos \left[\left(n_{0 d}-\frac{1}{2}\right) \frac{\pi k_{d}}{N_{d}}\right]\right\} \\
& \times\left\{\sum_{i=1}^{d} q_{i}\left[1-\cos \left(\frac{\pi k_{i}}{N_{i}}\right)\right]\right\}^{-1},
\end{aligned}
$$

the pseudo-Green function for reflecting domains $H\left(\vec{n} \mid \vec{n}_{0}\right)$ in $d$ dimensions and for arbitrary $q_{i}\left(0<q_{i} \leq 1\right)$ is given by

$\frac{H\left(\vec{n} \mid \overrightarrow{n_{0}}\right)}{d}=\left[\sum_{i=1}^{d} 2^{i} \sum_{\vec{k}}^{\vec{N}} \sum_{\vec{I} \in M_{i}} \mathcal{H}^{(r)}(\vec{I} \cdot \vec{k})\right]\left(\prod_{i=1}^{d} N_{i}\right)^{-1}$.

A similar analysis for the periodic lattices shows that

$\frac{H\left(\vec{n} \mid \overrightarrow{n_{0}}\right)}{d}=\left[\sum_{i=1}^{d} \sum_{\vec{k}}^{\vec{N}} \sum_{\vec{I} \in M_{i}} \mathcal{H}^{(p)}(\vec{I} \cdot \vec{k})\right]\left(\prod_{i=1}^{d} N_{i}\right)^{-1}$,

with

$\mathcal{H}^{(p)}(\vec{k})=\frac{\cos \left[\left(n_{1}-n_{01}\right) \frac{2 \pi k_{1}}{N_{1}}\right] \ldots \cos \left[\left(n_{d}-n_{0 d}\right) \frac{2 \pi k_{d}}{N_{d}}\right]}{\sum_{i=1}^{d} q_{i}\left[1-\cos \left(\frac{2 \pi k_{i}}{N_{i}}\right)\right]}$.

\section{FORMALISM FOR FIRST-PASSAGE PROCESSES WITH MULTIPLE TARGETS}

\section{A. Relation between propagator and first-passage probability to either of two targets}

The construction of the first-passage probability to either of two targets proceeds through a stepwise increase of the number of targets. I start from the case of two targets by finding a relation between the first-passage probability $G_{\vec{n}_{0}}^{(1)}\left(\vec{n}_{1}, t \mid \vec{n}_{2}\right)$ to reach $\vec{n}_{1}$ starting from $\vec{n}_{0}$ at time $t$ and not having reached $\vec{n}_{2}$ (between time 0 and time $t$ ), and the first-passage probability $F_{\vec{n}_{0}}\left(\vec{n}_{2}, t\right)$ to reach $\vec{n}_{2}$ at time $t$ starting from $\vec{n}_{0}$. The superscript (1) for $G$ indicates that only one lattice site is not reached - the superscript would be (2) if two lattice sites were not reached, and so on. For two targets, one can write the following relation [100]:

$$
\begin{aligned}
G_{\vec{n}_{0}}^{(1)} & \left(\vec{n}_{2}, t \mid \vec{n}_{1}\right) \\
& =F_{\vec{n}_{0}}\left(\vec{n}_{2}, t\right)-\sum_{t^{\prime}=0}^{t} G_{\vec{n}_{0}}^{(1)}\left(\vec{n}_{1}, t^{\prime} \mid \vec{n}_{2}\right) F_{\vec{n}_{1}}\left(\vec{n}_{2}, t-t^{\prime}\right) .
\end{aligned}
$$

Notice that $G_{\vec{n}_{0}}^{(1)}\left(\vec{n}_{2}, t \mid \vec{n}_{1}\right)$ is not normalized, as one realizes by summing Eq. (37) over all times. To construct the normalized conditional probability, one needs to consider $G_{\vec{n}_{0}}^{(1)}\left(\vec{n}_{2}, t \mid \vec{n}_{1}\right) / \mathcal{G}_{\vec{n}_{0}}^{(1)}\left(\vec{n}_{2}, z=1 \mid \vec{n}_{1}\right)$; that is, one needs to divide $G^{(1)}$ by the splitting probability of reaching $\vec{n}_{2}$ and not ever going to $\vec{n}_{1}$.

The generating function of Eq. (37) and of the alternative equation with $\vec{n}_{2}$ exchanged with $\vec{n}_{1}$ can be used jointly to obtain $\mathcal{G}_{\vec{n}_{0}}^{(1)}\left(\vec{n}_{2}, z \mid \vec{n}_{1}\right)$ and $\mathcal{G}_{\vec{n}_{0}}^{(1)}\left(\vec{n}_{1}, z \mid \vec{n}_{2}\right)$ and, from them, the first-passage probability in the $z$ domain to either target,

$$
\begin{aligned}
& \tilde{\mathcal{E}}_{\vec{n}_{0} \rightarrow\left(\vec{n}_{1} ; \vec{n}_{2}\right)}(z) \\
& =\left\{\tilde{F}_{\vec{n}_{0}}\left(\vec{n}_{1}, z\right)\left[1-\tilde{F}_{\vec{n}_{1}}\left(\vec{n}_{2}, z\right)\right]+\tilde{F}_{\vec{n}_{0}}\left(\vec{n}_{2}, z\right)\right. \\
& \left.\quad \times\left[1-\tilde{F}_{\vec{n}_{2}}\left(\vec{n}_{1}, z\right)\right]\right\}\left[1-\tilde{F}_{\vec{n}_{2}}\left(\vec{n}_{1}, z\right) \tilde{F}_{\vec{n}_{1}}\left(\vec{n}_{2}, z\right)\right]^{-1} .
\end{aligned}
$$

After a numerical $z$ inversion of Eq. (38), I plot in Fig. 6 the time-dependent first-passage probability to either target, namely, $\mathcal{E}_{\vec{n}_{0} \rightarrow\left(\vec{n}_{1} ; \vec{n}_{2}\right)}(t)$, for all possible $\vec{n}_{0}$. When $\vec{n}_{0} \neq \vec{n}_{1}, \vec{n}_{2}$, the data represent the first-passage probability, whereas when $\vec{n}_{0}$ coincides with the target at $\vec{n}_{1}$ or $\vec{n}_{2}$, the values at those points represent the probability, starting from $\vec{n}_{0}$, to either return to $\vec{n}_{0}$ or reach the other target. In other words, $G_{\vec{n}_{0}}^{(1)}\left(\vec{n}_{0}, t \mid \vec{n}_{2}\right)+G_{\vec{n}_{0}}^{(1)}\left(\vec{n}_{2}, t \mid \vec{n}_{0}\right)$ and $G_{\vec{n}_{0}}^{(1)}\left(\vec{n}_{0}, t \mid \vec{n}_{1}\right)+G_{\vec{n}_{0}}^{(1)}\left(\vec{n}_{1}, t \mid \vec{n}_{0}\right)$.

In Fig. 6, the complex structure of the surface plot is due to the locations near the targets having the first-passage probability already past their maximum value, compared to the lattice sites further away from the targets for which the first-passage probability has barely increased from its initial zero value. In addition, the marked asymmetry in the surface plot, when comparing lattice sites around each of the two targets, is due to the higher chance of staying at the boundaries near lattice site $(2,9)$.

Expression (38) can be exploited to derive other relevant quantities such as the MFPT to either of two targets via Eq. (13) to get

$$
\begin{aligned}
& T_{\vec{n}_{0} \rightarrow\left(\vec{n}_{1} ; \vec{n}_{2}\right)} \\
& =\frac{T_{\vec{n}_{0} \rightarrow \vec{n}_{1}} T_{\vec{n}_{1} \rightarrow \vec{n}_{2}}+T_{\vec{n}_{0} \rightarrow \vec{n}_{2}} T_{\vec{n}_{2} \rightarrow \vec{n}_{1}}-T_{\vec{n}_{1} \rightarrow \vec{n}_{2}} T_{\vec{n}_{2} \rightarrow \vec{n}_{1}}}{T_{\vec{n}_{1} \rightarrow \vec{n}_{2}}+T_{\vec{n}_{2} \rightarrow \vec{n}_{1}}},
\end{aligned}
$$




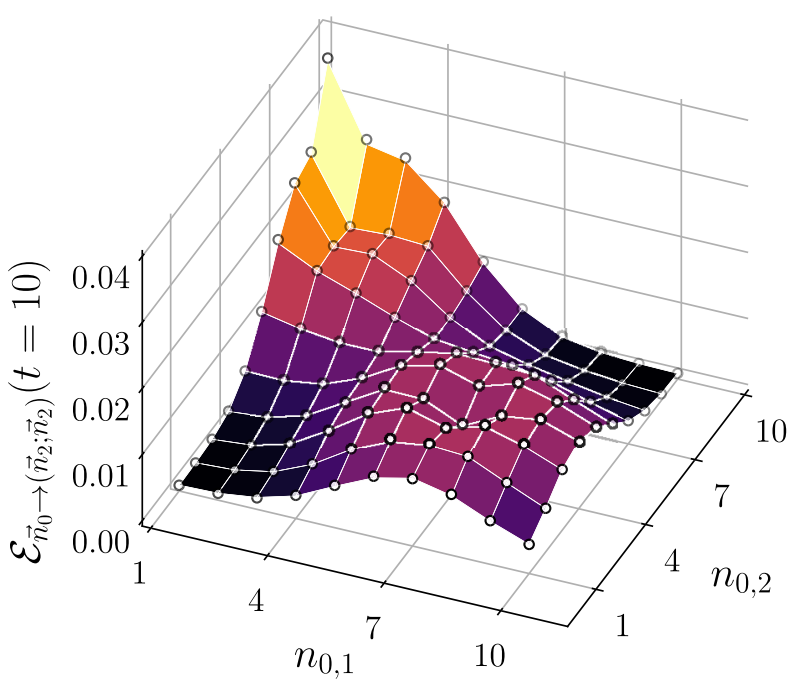

FIG. 6. First-passage probability $\mathcal{E}_{\vec{n}_{0} \rightarrow\left(\vec{n}_{1} ; \vec{n}_{2}\right)}(t)$ from any lattice site $\vec{n}_{0}$ to either of two targets, located at $(2,9)$ and $(7,4)$, in a square domain with reflecting boundaries of size $N=10$ at time $t=10$. At the location of the targets, the plot represents the return probability to the starting lattice without having been to the other target. The choice $q_{1}=q_{2}=1 / 3$ has been selected for the propagator $P_{\vec{n}_{0}}\left(n_{1}, n_{2}, t\right)$ in Eq. (21). The open white circles represent the numerical inversion of Eq. (38), while the shaded surface represents the ensemble average of $10^{6}$ stochastic simulations.

or the splitting probability to eventually reach $\vec{n}_{2}$ without having reached $\vec{n}_{1}$,

$$
\mathcal{G}_{\vec{n}_{0}}^{(1)}\left(\vec{n}_{2}, z=1 \mid \vec{n}_{1}\right)=\frac{T_{\vec{n}_{0} \rightarrow \vec{n}_{1}}+T_{\vec{n}_{1} \rightarrow \vec{n}_{2}}-T_{\vec{n}_{0} \rightarrow \vec{n}_{2}}}{T_{\vec{n}_{1} \rightarrow \vec{n}_{2}}+T_{\vec{n}_{2} \rightarrow \vec{n}_{1}}},
$$

and its counterpart $\mathcal{G}_{\vec{n}_{0}}^{(1)}\left(\vec{n}_{1}, z=1 \mid \vec{n}_{2}\right)$. Equations (39) and (40) match past results presented in Refs. [101,102].

\section{B. First-passage probability to either of multiple targets}

To construct the first-passage probability to either of a larger number of targets, one has to proceed stepwise. For three targets at $\vec{n}_{i}, \vec{n}_{j}$, and $\vec{n}_{k}$, one writes a relation between $G^{(1)}$ and $G^{(2)}$, namely,

$G_{\vec{n}_{0}}^{(2)}\left(\vec{n}_{i}, t \mid \vec{n}_{j}, \vec{n}_{k}\right)$

$=G_{\vec{n}_{0}}^{(1)}\left(\vec{n}_{i}, t \mid \vec{n}_{k}\right)-\sum_{t^{\prime}=0}^{t} G_{\vec{n}_{0}}^{(2)}\left(\vec{n}_{j}, t^{\prime} \mid \vec{n}_{i}, \vec{n}_{k}\right) G_{\vec{n}_{j}}^{(1)}\left(\vec{n}_{i}, t-t^{\prime} \mid \vec{n}_{k}\right)$,

which gives the first-passage probability of reaching $\vec{n}_{i}$ and not having reached $\vec{n}_{j}$ or $\vec{n}_{k}$. The first term of $G_{\vec{n}_{0}}^{(2)}\left(\vec{n}_{i}, t \mid \vec{n}_{j}, \vec{n}_{k}\right)$ is given by the first-passage probability $G_{\vec{n}_{0}}^{(1)}\left(\vec{n}_{i}, t \mid \vec{n}_{k}\right)$ given in Eq. (37) of having reached $\vec{n}_{i}$ at time $t$ and not $\vec{n}_{k}$ (but with no constraints on the number of times the walker has gone through $\vec{n}_{j}$ ) minus the contribution of having reached $\vec{n}_{j}$ at some earlier time $t^{\prime}$ and never $\vec{n}_{k}$ and $\vec{n}_{i}$, and subsequently, in the time $t-t^{\prime}$ having reached $\vec{n}_{i}$ and not $\vec{n}_{k}$. By additionally writing $G_{\vec{n}_{0}}^{(2)}\left(\vec{n}_{i}, t \mid \vec{n}_{j}, \vec{n}_{k}\right)$ with $\vec{n}_{i}, \vec{n}_{j}$, and $\vec{n}_{k}$ permuted, it is possible to derive the analytic expression for the generating function of the first-passage probability $\tilde{\mathcal{E}}_{\vec{n}_{0} \rightarrow\left(\vec{n}_{i} ; \vec{n}_{j} ; \vec{n}_{k}\right)}(z)$ to one of three targets [see Eq. (S21) in Ref. [75] ].

The procedure shown for the three-target case can be extended to an arbitrary number $m$ of targets by writing an equation analogous to Eq. (41) that links $G^{(m-1)}$ to $G^{(m-2)}$. Although the procedure to build the relations is tedious because it requires one to write a number of equations equal to the number of permutations of the $m-1$ targets in the $G^{(m-1)}$ equation, it is possible, in principle, to construct the general first-passage probability to either of the $m$ targets.

\section{Mean first-passage time to either of multiple targets}

I apply the general formalism above to find the MFPT to either of a set of targets. The construction of such MFPT expressions represents an alternative to the use of the pseudo-Green function formalism for which the multitarget MFPT is expressed by seeking a solution of a set of $m$ simultaneous equations for the splitting probabilities and

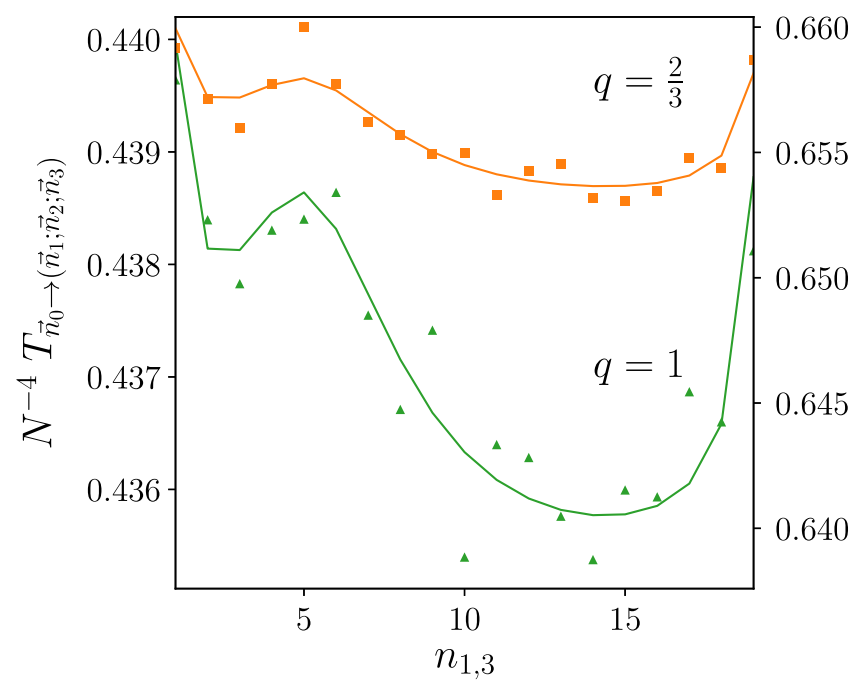

FIG. 7. Mean first-passage time in a 4D domain with reflecting boundaries of size $N_{i}=21$, with $i=1,2,3,4$, to either of three targets as a function of the $n_{1,1}$ coordinate. The walker initial position is at $\vec{n}_{0}=(5,4,2,1)$. The coordinates of the first target are $\vec{n}_{1}=\left(n_{1,1}, 8,9,9\right)$, with $2 \leq n_{1,1} \leq 20$, while the second and third targets are fixed at $\vec{n}_{2}=(8,2,1,10)$ and $\vec{n}_{3}=(6,5,8,7)$. The lines are obtained using the MFPT to either of three targets displayed in Eq. (S24) in Ref. [75], with the MFPT between any two lattice sites provided by Eq. (31), while the dots are obtained by averaging $10^{6}$ stochastic simulations. 
the pairwise pseudo-Green functions [see Eqs. (25) and (26) in Ref. [103] ].

I study, in particular, the case of three targets, as for a larger number of targets, the expressions are quite imposing. Using $\tilde{\mathcal{E}}_{\vec{n}_{0} \rightarrow\left(\vec{n}_{i} ; \vec{n}_{i} ; \vec{n}_{k}\right)}(z)$, I obtain the MFPT, $T_{\vec{n}_{0} \rightarrow\left(\vec{n}_{i} ; \vec{n}_{i} ; \vec{n}_{k}\right)}$, to either of three targets in arbitrary dimensions [see Eq. (S24) in Ref. [75] ]. I study the case of a 4D domain with reflective boundaries with two targets fixed; in Fig. 7 I show the MFPT as a function of the coordinate of the third target along one axis and for different values of the probability of staying at a site, $q_{i}$, set equal to $q$ for each $i$. When $q_{i}=q$ the MFPT to either target has a trivial $q^{-1}$ dependence.

\section{CONCLUSIONS}

The diffusion equation is one of a small set of fundamental equations that has left a legacy across a vast number of disciplines. The version where space and time are continuous variables is commonly used and has been solved exactly in unbounded and bounded domains in arbitrary dimensions. Surprisingly, the same could not be said about the space-time discrete diffusion equation. While closed-form expressions are known for unbounded $d$-dimensional space, in finite $d$-dimensional domains, propagators are only known for periodic domains and for some $1 \mathrm{~d}$ cases, e.g., absorbing boundaries. The generating function and the time-dependent expression for arbitrary boundary conditions and arbitrary domains have, until now, remained an outstanding problem.

The work presented here has a brought a resolution to this outstanding problem by finding the analytic form of the LRW propagator in various bounded domains, namely, reflecting boundaries, periodic boundaries, absorbing boundaries, and a mixed scenario with one reflecting and one absorbing boundary. Given the vast applicability of the diffusion equation, the findings in this work represent a fundamental contribution to the arsenal of tools that are consistently employed to analyze and predict random processes across scales and disciplines.

The exact form of the confined LRW propagator bypasses the need to construct and seek the solution of a master equation, and it allows one to quantify the time dependence of a variety of transport processes. Quantities such as the first-passage probability, return probability, and average number of distinct sites, which were mainly accessible via stochastic simulations or numerical techniques $[99,104,105]$, can now be obtained analytically in 1D or through a numerical inversion of the associated generating function in higher dimensions.

With the analytic expression of the propagator generating function, it has also been possible to straightforwardly derive the MFPT to a single target in arbitrary dimensions. The sought-after MFPT formulas have allowed me to find the discrete pseudo-Green functions for reflecting and periodic domains in arbitrary dimensions, going beyond the formulas known so far only up to 3D. A procedure to analytically determine the first-passage probability and the MFPT with multiple targets (and arbitrary dimensions) has also been presented. Although it has been discussed in the context of confined Euclidean lattices, it has general validity. It is thus applicable to study LRW in other types of geometries as well as in networks [106].

By relaxing certain assumptions of the statistical features of the walk, one can study cases other than the symmetric nearest-neighbor LRW that I have studied here. It is, in fact, possible to derive analytic representations of the propagators for next-nearest-neighbor and biased LRW in confined lattices of arbitrary dimensions, and one may also attempt to analytically derive the propagator for $1 \mathrm{D}$ and $2 \mathrm{D}$ correlated LRW [107] in confined domains for which, so far, the MFPT has been the main focus of the analyses in Refs. [108,109]. Extensions to situations where the lattice is not Euclidean (e.g., a triangular lattice in 2D [26]), when reflection at a boundary is only partial [110], and, more generally, to disordered lattices are also interesting future directions.

Of relevance to a plethora of empirical situations is a general theory of transport in disordered lattices [18] whereby the spatial disorder is replaced by a temporal memory [111] transforming the Markov master equation representing the movement of the walk to a non-Markov description in the form of a generalized master equation (in discrete time) $[112,113]$. As the key ingredient to develop such a theory is knowledge of the analytic expression of the propagator generating function, an effective medium theory of LRW in finite disordered lattices is within reach.

Potential future directions include the study of other statistical features of the walk (which so far has been tackled only for translationally invariant systems such as the expected lattice random walk maximum [114]), and the analysis of meanderers, bridges, excursions [115], and record statistics $[116,117]$. With the help of the present findings, more complex movement processes in confined space could also be studied, including resetting walks [118-122], self-avoiding walks [26], loop-erased walks [123], and reinforced walks [53,124].

It is also worth citing the possibility to analyze various quantities that have already been studied in the past for confined lattices and that could be further explored. They include occupation times [125], mortal walkers and killing times [26], narrow escape times [126-128], cover times $[129,130]$, and encounter times $[131,132]$. Moreover, some important models of movement in confined space where a walker interacts with its environments or with other individuals, e.g., a bias towards a focal point [31,133] or the transmission of an infection [134,135], have surprisingly been studied mainly via Brownian walks, despite being perfectly suited for LRW.

The formalism presented here could also be used as a starting point for many-body problems such as in the 
presence of excluded volume interactions, e.g., single-file dynamics in finite lattices for which the occupation probability in continuous space and time is known analytically [136,137].

For walks in continuous time but discrete space, the formalism developed in Sec. V C connects to a wide body of literature on continuous time random walks [138-141], which naturally allows one to extend the findings presented here to situations in which the walker displays anomalous diffusive characteristics. The present work could be used to study phenomena such as ergodicity breaking [142,143] and its connection to fractional diffusion $[144,145]$ in the context of bounded domains.

I conclude by pointing to the fact that the existence of different analytic expressions in 1D to represent the propagator generating function, through either a finite series with trigonometric functions or its sum, allows one to generate various trigonometric identities. Such identities appear to be absent from the literature on analytic combinatorics [58,146], Chebyshev polynomials [147,148], or finite trigonometric sums [149-154]. While I have presented a few examples of such identities in this paper, including the ones necessary to construct the propagators in higher dimensions, there are various others that can be deduced.

\section{ACKNOWLEDGMENTS}

I acknowledge discussions with Eli Barkai, Oliviér Benichou, Denis Boyer, Nello Cristianini, Shamik Gupta, Mike Jeffrey, Rainer Klages, Satya Majumdar, Ralf Metzler, and David Sanders. I am thankful for the support of EPSRC Grant No. EP/I013717/1, and I am indebted to Alastair Franklin and Seeralan Sarvaharman for help with creating the figures. All underlying data are provided within this paper and the accompanying Supplemental Material.

\section{APPENDIX A: PROPAGATOR IN 1D INFINITE DOMAIN}

The Fourier transform solution of Eq. (1) is $\hat{\mathcal{Q}}(\kappa, t)=$ $\hat{\mathcal{Q}}(\kappa, 0)[1-q+q \cos (\kappa)]^{t}$, where $\hat{\mathcal{Q}}(\kappa, t)=\sum_{n=-\infty}^{+\infty} e^{-i \kappa n} \times$ $\mathcal{Q}(n, t)$ with its generating function $\tilde{\hat{\mathcal{Q}}}(\kappa, z)=\hat{\mathcal{Q}}(\kappa, 0) \times$ $\{1-z[1-q+q \cos (\kappa)]\}^{-1}$. The inverse Fourier transformation,

$$
\tilde{\mathcal{Q}}(n, z)=\frac{1}{2 \pi} \int_{-\pi}^{+\pi} d \kappa \frac{\cos \left[\left(n-n_{0}\right) \kappa\right]}{1-z[1-q+q \cos (\kappa)]},
$$

can be performed explicitly through the variable change $\kappa=\tan (x / 2)$ - and can also be found in Eq. (3.613) of Ref. [155]—resulting in

$$
\tilde{\mathcal{Q}}_{n_{0}}(n, z)=\frac{\left(\frac{1}{\beta(z)}+\sqrt{\frac{1}{\beta^{2}(z)}-1}\right)^{-\left|n-n_{0}\right|}}{[1-z(1-q)] \sqrt{1-\beta^{2}(z)}},
$$

where

$$
\beta(z)=\frac{z q}{1-z(1-q)}
$$

\section{APPENDIX B: METHOD OF IMAGES: 1D FINITE DOMAIN PROPAGATORS}

The method of images is employed in the reflecting and absorbing case by considering the contribution rom an infinite number of images as $\tilde{P}_{n_{0}}^{(r)}(n, z)=$ $\sum_{k=-\infty}^{+\infty}\left[\tilde{\mathcal{Q}}_{n_{0}+2 k N}(n, z)+\tilde{\mathcal{Q}}_{-n_{0}+1+2 k N}(n, z)\right]$ for the reflective case and $\tilde{P}_{n_{0}}^{(a)}(n, z)=\sum_{k=-\infty}^{+\infty}\left[\tilde{\mathcal{Q}}_{n_{0}+2 k(N-1)}(n, z)-\right.$ $\left.\tilde{\mathcal{Q}}_{-n_{0}+2+2 k(N-1)}(n, z)\right]$ for the absorbing case. For the domain with one reflective boundary and one absorbing boundary, I consider a domain of $2 N-1$ lattice sites with reflective boundaries and construct the propagator $\quad \tilde{P}_{n_{0}}^{(r, 2 N-1)}(n, z)=\sum_{k=-\infty}^{+\infty} \tilde{\mathcal{Q}}_{n_{0}+2 k(2 N-1)}(n, z)+$ $\tilde{\mathcal{Q}}_{-n_{0}+1+2 k(2 N-1)}(n, z)$, where the additional superscript $2 N-1$ is used to distinguish it from the propagator $P_{n_{0}}^{(r)}(n, z)$ that, by definition, has $1 \leq n \leq N$. I then employ the method of images for a single absorbing boundary located halfway at $n=N$ and obtain $\tilde{P}_{n_{0}}^{(m)}(n, z)=$ $\tilde{P}_{n_{0}}^{(r, 2 N-1)}(n, z)-\tilde{P}_{-n_{0}+2 N}^{(r, 2 N-1)}(n, z)$. Finally, for the periodic case, I simply wrap the infinite propagator onto itself over $N$ sites by taking $\tilde{P}_{n_{0}}^{(p)}(n, z)=\sum_{k=-\infty}^{+\infty} \widetilde{\mathcal{Q}}_{n_{0}+k N}(n, z)$. These technical steps give the various expressions in Eq. (2). Details of the derivations are given in Ref. [75].

\section{APPENDIX C: TIME DEPENDENCE OF 1D DERIVED QUANTITIES}

Here, I present the time dependence of the return probability, the first-passage probability to one target, and the average number of distinct sites visited for the reflecting and periodic cases. In order to do so, one has to rewrite the propagator with the help of Chebyshev polynomials [147] $\mathcal{T}_{n}(s), \mathcal{U}_{n}(s)$, and $\mathcal{V}_{n}(s)$, respectively, of the first, second, and third kinds (of order $n$ ). The propagator generating functions in Eq. (2) are rewritten using the relations $\mathcal{T}_{n}(s)=\cosh [n \operatorname{arcCosh}(s)], \mathcal{U}_{n}(s)=\sinh [n \operatorname{arcCosh}(s)] /$ $\sinh [\operatorname{arcCosh}(s)]$, and $\mathcal{V}_{n}(s)=\cosh [(n+1 / 2) \operatorname{arcCosh}(s)] /$ $\cosh [\operatorname{arcCosh}(s) / 2]$ for $|s| \geq 1$.

\section{First-passage probability with reflecting boundaries}

The generating function of the first-passage probability in Eq. (9) is rewritten as

$$
\tilde{F}_{n_{0}}^{(r)}(n, z)=\frac{\mathcal{V}_{N-n_{>}}\left(1+\frac{1}{q}\left[\frac{1}{z}-1\right]\right) \mathcal{V}_{n_{<}-1}\left(1+\frac{1}{q}\left[\frac{1}{z}-1\right]\right)}{\mathcal{V}_{N-n}\left(1+\frac{1}{q}\left[\frac{1}{z}-1\right]\right) \mathcal{V}_{n-1}\left(1+\frac{1}{q}\left[\frac{1}{z}-1\right]\right)}
$$


To obtain the time dependence, it can be conveniently split into two expressions depending on whether the target site is to the left or right of the initial position. With the factorization [156] $\mathcal{V}_{m}(x)=2^{m} \prod_{k=1}^{m}\{x-\cos [(2 k-1) \pi / 2 m+1]\}$, the calculation of the residues at the poles gives

$F_{n_{0}}^{(r)}(n, t)= \begin{cases}\sum_{m=1}^{n-1} \frac{2 q(-1)^{m+1}}{2 n-1} \cos \left[\frac{\left(2 n_{0}-1\right)(2 m-1)}{2 n-1} \frac{\pi}{2}\right]\left|\sin \left[\frac{2 m-1}{2 n-1} \pi\right]\right|\left[1-q+q \cos \left(\frac{2 m-1}{2 n-1} \pi\right)\right]^{t-1} & n>n_{0} \\ \sum_{m=1}^{N-n} \frac{2 q(-1)^{m+1}}{2(N-n)+1} \cos \left[\frac{\left\{2\left(N-n_{0}\right)+1\right\}(2 m-1)}{2(N-n)+1} \frac{\pi}{2}\right]\left|\sin \left[\frac{2 m-1}{2(N-n)+1} \pi\right]\right|\left[1-q+q \cos \left(\frac{2 m-1}{2(N-n)+1} \pi\right)\right]^{t-1} & n<n_{0},\end{cases}$

which is valid for $t \geq 1$, while one has $F_{n_{0}}(n, 0)=0$ for any $n \neq n_{0}$.

\section{First-passage probability with periodic boundaries}

The time dependence of the first-passage probability in the periodic case is conveniently derived by rewriting Eq. (11) as

$$
\tilde{F}_{n_{0}}^{(p)}(n, z)=\frac{\mathcal{T}_{N-\left|n-n_{0}\right|+1}\left(\frac{1}{\beta(z)}\right)-\mathcal{T}_{N-\left|n-n_{0}\right|-1}\left(\frac{1}{\beta(z)}\right)+\mathcal{T}_{\left|n-n_{0}\right|+1}\left(\frac{1}{\beta(z)}\right)-\mathcal{T}_{\left|n-n_{0}\right|-1}\left(\frac{1}{\beta(z)}\right)}{2\left(\frac{1}{\beta^{2}(z)}-1\right) \mathcal{U}_{N-1}\left(\frac{1}{\beta(z)}\right)},
$$

valid for any $n \neq n_{0}$. In this case, the factorized form $\mathcal{U}_{n}(\sigma)=2^{n} \prod_{k=1}^{n}\{\sigma-\cos [(\pi k) /(n+1)]\}$ allows me to calculate the residues at the poles. The inversion provides the sought-after expression for $t \geq 1$,

$$
F_{n_{0}}^{(p)}(n, t)=\frac{q}{N} \sum_{k=1}^{N-1}\left[1-(-1)^{k}\right] \sin \left[\frac{\left|n-n_{0}\right| \pi k}{N}\right] \sin \left[\frac{\pi k}{N}\right]\left[1-q+q \cos \left(\frac{\pi k}{N}\right)\right]^{t-1},
$$

with $F_{n_{0}}(n, 0)=0$.

\section{First-passage probability to either of two boundaries}

In the presence of two lattice sites where a walker may be absorbed, the first-passage probability to either of them is

$$
\mathcal{E}_{n_{0} \rightarrow(1 ; N)}(t)=\frac{q}{N-1} \sum_{k=1}^{N-2}\left[1-(-1)^{k}\right] \sin \left[\frac{n_{0}-1}{N-1} \pi k\right] \sin \left(\frac{\pi k}{N-1}\right)\left[1-q+q \cos \left(\frac{\pi k}{N-1}\right)\right]^{t-1},
$$

with $\mathcal{F}_{n_{0}}(n, 0)=0$. It is not surprising that the expression resembles the first-passage probability of a single absorbing lattice site in a periodic domain as the physics of the system is similar. By creating a symmetric initial location relative to the boundaries, the physics of the box and that of the periodic domain coincide. If one considers an initial walker location placed precisely in the middle of a domain of size $N+1$ with $N$ odd, Eq. (C5) is identical to the first-passage probability in a periodic domain of size $N$ when the separation between the initial location and the absorbing site in Eq. (C4) is $(N-1) / 2$.

\section{APPENDIX D: AVERAGE NUMBER OF DISTINCT SITES VISITED}

\section{Reflecting boundaries}

After some laborious algebra, the inversion of the generating function $\tilde{\mathcal{M}}_{n_{0}}^{(r)}(z)$ gives the explicit timedependent expression

$$
\begin{aligned}
\mathcal{M}_{n_{0}}^{(r)}(t)= & N-\frac{1}{2 n_{0}-1} \sum_{m=1}^{n_{0}-1} \frac{(-1)^{m+1} \sin \left(\frac{2 m-1}{2 n_{0}-1} N \pi\right) \cot ^{2}\left(\frac{2 m-1}{2 n_{0}-1} \frac{\pi}{2}\right)}{\cos \left[\frac{\left\{2\left(N-n_{0}\right)+1\right\}(2 m-1)}{2 n_{0}-1} \frac{\pi}{2}\right]}\left[1-q+q \cos \left(\frac{2 m-1}{2 n_{0}-1} \pi\right)\right]^{t} \\
& -\frac{1}{2\left(N-n_{0}\right)+1} \sum_{m=1}^{N-n_{0}} \frac{(-1)^{m+1} \sin \left(\frac{2 m-1}{2\left(N-n_{0}\right)+1} N \pi\right) \cot ^{2}\left(\frac{2 m-1}{2\left(N-n_{0}\right)+1} \frac{\pi}{2}\right)}{\cos \left[\frac{\left\{2 n_{0}-1\right\}(2 m-1)}{2\left(N-n_{0}\right)+1} \frac{\pi}{2}\right]}\left[1-q+q \cos \left(\frac{2 m-1}{2\left(N-n_{0}\right)+1} \pi\right)\right]^{t},
\end{aligned}
$$

which implicitly assumes that when $n_{0}=1$ the first summation is absent, and the same occurs for the second summation when $n_{0}=N$. By verifying that indeed $\mathcal{M}_{n_{0}}^{(r)}(0)=1$, one derives an intricate trigonometric identity 


$$
\sum_{m=1}^{x} \frac{(-1)^{m+1} \sin \left(\frac{2 m-1}{2 x+1} N \pi\right) \cot ^{2}\left(\frac{2 m-1}{2 x+1} \frac{\pi}{2}\right)}{\cos \left[\frac{\{2(N-x)-1\}(2 m-1)}{2 x+1} \frac{\pi}{2}\right]}=(2 x+1) x,
$$

where $x$ represents an integer that is smaller than or equal to $N$.

\section{Periodic boundaries}

As expected, the translational invariance gives a time dependence for the mean number of distinct visited sites independent of the initial position, namely,

$$
\begin{aligned}
\mathcal{M}^{(p)}(t)= & N-\frac{1}{N} \sum_{k=1}^{N-1} \frac{\left[1-(-1)^{k}\right]\left[1+\cos \left(\frac{\pi k}{N}\right)\right]}{1-\cos \left(\frac{\pi k}{N}\right)} \\
& \times\left[1-q+q \cos \left(\frac{\pi k}{N}\right)\right]^{t} .
\end{aligned}
$$

After rewriting $(-1)^{k}=\cos (\pi k / N)$, the identity (E2) below allows one to verify that $\mathcal{M}^{(p)}(t=0)=1$.

\section{APPENDIX E: FINITE-SERIES IDENTITIES WITH CHEBYSHEV POLYNOMIALS}

I derive a set of three finite-series identities that are necessary to construct the propagator in dimensions larger than 1 . Each identity relates to the four boundary conditions and is valid for integers $m$ and for any complex value $\sigma$. For the reflecting and absorbing case, I use the relation, valid for $|m| \leq 2 N$,

$$
\begin{aligned}
\sum_{k=1}^{N-1} \frac{\cos \left(\frac{\pi m k}{N}\right)}{\sigma-\cos \left(\frac{\pi k}{N}\right)}= & N \frac{\mathcal{T}_{|N-| m||}(\sigma)}{\left(\sigma^{2}-1\right) \mathcal{U}_{N-1}(\sigma)} \\
& +\frac{1}{2}\left[\frac{1}{1-\sigma}+\frac{(-1)^{m+1}}{1+\sigma}\right],
\end{aligned}
$$

with $\mathcal{T}_{n}(\sigma)$ and $\mathcal{U}_{n}(\sigma)$ Chebyshev polynomials, respectively, of the first and second kinds, of order $n$. In the limit $\sigma \rightarrow 1$, using the fact that $\mathcal{T}_{n}(1)=1, \mathcal{U}_{n}(1)=n+1$, $\mathcal{T}_{n}^{\prime}(1)=n^{2}, \mathcal{U}_{n}^{\prime}(1)=n\left(n^{2}+3 n+2\right) / 3$, one has

$$
\begin{aligned}
\sum_{k=1}^{N-1} \frac{\cos \left(\frac{\pi m k}{N}\right)}{1-\cos \left(\frac{\pi k}{N}\right)}= & \frac{1}{3}\left(N^{2}+\frac{1}{2}\right)+|m|\left(\frac{|m|}{2}-N\right) \\
& +\frac{(-1)^{m+1}-1}{4} .
\end{aligned}
$$

For the periodic case, I employ

$\sum_{k=1}^{N-1} \frac{\cos \left(\frac{2 \pi m k}{N}\right)}{\sigma-\cos \left(\frac{2 \pi k}{N}\right)}=\frac{1}{1-\sigma}+N \frac{\mathcal{T}_{N-|m|}(\sigma)+\mathcal{T}_{|m|}(\sigma)}{\left(\sigma^{2}-1\right) \mathcal{U}_{N-1}(\sigma)}$

which is valid for $|m| \leq N$ and has the limit

$$
\sum_{k=1}^{N-1} \frac{\cos \left(\frac{2 \pi m k}{N}\right)}{1-\cos \left(\frac{2 \pi k}{N}\right)}=\frac{1}{6}\left(N^{2}-1\right)+|m|(|m|-N) .
$$

For the mixed case, I have derived the identity

$$
\begin{aligned}
& \sum_{k=1}^{N-1} \frac{\cos \left(\frac{2 k-1}{2 N-1} m \pi\right)}{\sigma-\cos \left(\frac{2 k-1}{2 N-1} \pi\right)}=\frac{1}{2} \frac{(-1)^{m+1}}{1+\sigma} \\
& \quad+\left(N-\frac{1}{2}\right) \frac{\mathcal{T}_{2 N-1-|m|}(\sigma)-\mathcal{T}_{|m|}(\sigma)}{\left(\sigma^{2}-1\right) \mathcal{U}_{2 N-2}(\sigma)}
\end{aligned}
$$

with $|m| \leq 2 N-1$. Once again using the properties of the Chebyshev polynomials with their argument evaluated at 1 , that is, when $\sigma \rightarrow 1$, an additional relation similar to the limiting cases in Eqs. (E2) and (E4) can be derived.

Equations (E1), (E3), and (E5) are generalizations of known trigonometric identities (see, e.g., Ref. [157]). They can be obtained as a partial fraction expansion or, more generally, by identifying the residues and performing a complex contour integration that gives $f(\sigma)=$ $\sum_{i} \operatorname{Res}\left\{f(s) /(\sigma-s), s_{i}\right\}$, where $s_{i}$ are the poles of $f(s)$ [158]. Taking $f(s)$ as the right-hand sides of Eqs. (E1), (E3), and (E5), one can compute the residue contribution as $f(s)$ has poles of order one that can be located analytically due to the factorized form of $\mathcal{U}_{n}(\sigma)$. Note that the blowups on the right-hand sides of Eqs. (E1), (E3), and (E5) at $\sigma= \pm 1$ are only apparent as they result from removable singularities.

[1] I. Todhunter, A History of the Mathematical Theory of Probability from the Time of Pascal to that of Laplace (Cambridge University Press, Cambridge, England, 2016).

[2] K. Pearson, The Problem of the Random Walk, Nature (London) 72, 342 (1905).

[3] L. Bachelier, Theorie de la Speculation, Doctoral Thesis, Annales Scientifiques Ecole Normale Superieure III-17, 1900.

[4] L. Bachelier, M. Davis, and A. Etheridge, Louis Bachelier's Theory of Speculation: The Origins of Modern Finance (Princeton University Press, Princeton, NJ, 2011).

[5] M. von Smoluchowski, Essai d'une Théorie Cinétique du Mouvement Brownien et des Milieux Troubles, Bull. Int. Acad. Sci. Cracovie 7, 577 (1906).

[6] A. Einstein, Über die von der Molekularkinetischen Theorie der Wärme Geforderte Bewegung von in Ruhenden Flüssigkeiten Suspendierten Teilchen, Ann. Phys. (Berlin) 322, 549 (1905).

[7] M. von Smoluchowski, Sur le Chemin Moyen Parcouru par les Molecules d'un Gaz et sur son Rapport avec la Théorie de la Diffusion, Bull. Int. Acad. Sci. Cracovie 7, 202 (1906).

[8] M. von Smoluchowski, Zur Kinetischen Theorie der Brownschen Molekular Bewegung und der Suspensionen, Ann. Phys. (Berlin) 326, 756 (1906). 
[9] S. Chandrasekhar, Stochastic Problems in Physics and Astronomy, Rev. Mod. Phys. 15, 1 (1943).

[10] M. C. Wang and G. E. Uhlenbeck, On the Theory of the Brownian Motion II, Rev. Mod. Phys. 17, 323 (1945).

[11] M. Kac, On the Notion of Recurrence in Discrete Stochastic Processes, Bull. Am. Math. Soc. 53, 1002 (1947).

[12] S. Chandrasekhar, M. Kac, and R. Smoluchowski, Marian Smoluchowski, His Life and Scientific Work (Polish Scientific Publishers, Warszawa, 1986).

[13] W. Ebeling, E. Gudowska-Nowak, and I. M. Sokolov, On Stochastic Dynamics in Physics-Remarks on History and Terminology, Acta Phys. Pol. B 39, 1003 (2008).

[14] A. A. Markov, Extension of the Limit Theorems of Probability Theory to a Sum of Variables Connected in a Chain, edited by R. A. Howard, in Dynamic Probabilistic Systems: Markov Models (Wiley, New York, 1971), Vol. 1, pp. 552-576.

[15] G. Pólya, Quelques Problemes de Probabilité se Rapportant a la Promenade au Hasard?, L'Enseignement Mathématique 20, 8 (1919).

[16] G. Pólya, Über eine Aufgabe der Wahrscheinlichkeitsrechnung Betreffend die Irrfahrt im Straßennetz, Math. Ann. 84, 149 (1921).

[17] V. M. Kenkre and P. Reineker, Exciton Dynamics in Molecular Crystals and Aggregates, Springer Tracts in Modern Physics (Springer, Berlin, 1982), Vol. 94.

[18] B. D. Hughes, Random Walks and Random Environments: Random Walks (Clarendon Press, Oxford, 1995), Vol. 2.

[19] E. W. Montroll and G. H. Weiss, Random Walks on Lattices II, J. Math. Phys. (N.Y.) 6, 167 (1965).

[20] E. W. Montroll, Random Walks on Lattices. III. Calculation of First-Passage Times with Application to Exciton Trapping on Photosynthetic Units, J. Math. Phys. (N.Y.) 10, 753 (1969).

[21] V. M. Kenkre and Y. M. Wong, Theory of Exciton Migration Experiments with Imperfectly Absorbing End Detectors, Phys. Rev. B 22, 5716 (1980).

[22] W. T. F. Den Hollander and P. W. Kasteleyn, Random Walks with Spontaneous Emission on Lattices with Periodically Distributed Imperfect Traps, Physica A 112, 523 (1982).

[23] S. Havlin, G. H. Weiss, J. E. Kiefer, and M. Dishon, Exact Enumeration of Random Walks with Traps, J. Phys. A 17, L347 (1984).

[24] E. W. Montroll and B. J. West, On an Enriched Collection of Stochastic Processes, Studies in Statistical Mechanics: Fluctuation Phenomena, edited by E. W. Montroll and J. J. Lebowitz (North Holland, Amsterdam, 1979), Vol. VII, pp. 61-175.

[25] G. H. Weiss, Aspects and Applications of the Random Walk (North Holland, Amsterdam, 1994).

[26] B. D. Hughes, Random Walks and Random Environments: Random Walks (Clarendon Press, Oxford, 1995), Vol. 1.

[27] A. Okubo, Diffusion and Ecological Problems: Mathematical Models, in Biomathematics (Springer-Verlag, Berlin, 1980), Vol. 10.

[28] P. M. Kareiva and N. Shigesada, Analyzing Insect Movement as a Correlated Random Walk, Oecologia 56, 234 (1983).
[29] A. Okubo and S. A. Levin, Diffusion and Ecological Problems: Modern Perspectives, 2nd ed. (Springer-Verlag, New York, 2001).

[30] G. M. Viswanathan, M. G. E. da Luz, E. P. Raposo, and H. E. Stanley, The Physics of Foraging: An Introduction to Random Searches and Biological Encounters (Cambridge University Press, Cambridge, England, 2011).

[31] L. Giuggioli and V. M. Kenkre, Consequences of Animal Interactions on Their Dynamics: Emergence of Home Ranges and Territoriality, Mov. Ecol. 2, 20 (2014).

[32] H. C. Berg and D. A. Brown, Chemotaxis in Escherichia Coli Analysed by Three-Dimensional Tracking, Nature (London) 239, 500 (1972).

[33] H. G. Othmer, S. R. Dunbar, and W. Alt, Models of Dispersal in Biological Systems, J. Math. Biol. 26, 263 (1988).

[34] H. C. Berg, Random Walks in Biology (Princeton University, Princeton, NJ, 1993).

[35] D. Boal, Mechanics of the Cell (Cambridge University Press, Cambridge, England, 2012).

[36] O. Pulkkinen and R. Metzler, Distance Matters: The Impact of Gene Proximity in Bacterial Gene Regulation, Phys. Rev. Lett. 110, 198101 (2013).

[37] M. E. J. Newman, A Measure of Betweenness Centrality Based on Random Walks, Soc. Networks 27, 39 (2005).

[38] E. Scalas, Five Years of Continuous-Time Random Walks in Econophysics, in The Complex Networks of Economic Interactions, edited by A. Namatame, T. Kaizouji, and Y. Aruka (Springer, New York, 2006), pp. 3-16.

[39] B. D. Hughes, M. F. Shlesinger, and E. W. Montroll, Random Walks with Self-Similar Clusters, Proc. Natl. Acad. Sci. USA 78, 3287 (1981).

[40] R. Gorenflo, G. De Fabritiis, and F. Mainardi, Discrete Random Walk Models for Symmetric Lévy-Feller Diffusion Processes, Physica A 269, 79 (1999).

[41] D. Ben-Avraham and S. Havlin, Diffusion and Reactions in Fractals and Disordered Systems (Cambridge University Press, Cambridge, England, 2000).

[42] D. Sornette, Critical Phenomena in Natural Sciences: Chaos, Fractals, Self-Organization and Disorder: Concepts and Tools (Springer, Berlin, 2006).

[43] S. Redner, A Guide to First-Passage Processes (Cambridge University Press, Cambridge, England, 2001).

[44] A. J. Bray, S. N. Majumdar, and G. Schehr, Persistence and First-Passage Properties in Nonequilibrium Systems, Adv. Phys. 62, 225 (2013).

[45] O. Bénichou and R. Voituriez, From First-Passage Times of Random Walks in Confinement to Geometry-Controlled Kinetics, Phys. Rep. 539, 225 (2014).

[46] S. Redner, R. Metzler, and G. Oshanin, First-Passage Phenomena and Their Applications (World Scientific, Singapore, 2014).

[47] O. Bénichou, P. Illien, G. Oshanin, A. Sarracino, and R. Voituriez, Tracer Diffusion in Crowded Narrow Channels, J. Phys. Cond. Mat. 30, 443001 (2018).

[48] S. Ouvry and A. P. Polychronakos, Exclusion statistics and lattice random walks, Nucl. Phys. B., 948, 114731 (2019).

[49] T. M. Michelitsch, B. A. Collet, A. P. Riascos, A. F. Nowakowski, and F. C. G. A. Nicolleau, Fractional Random Walk Lattice Dynamics, J. Phys. A 50, 055003 (2017). 
[50] G. Schehr and S. N. Majumdar, Exact Record and Order Statistics of Random Walks via First-Passage Ideas, First-Passage Phenomena and Their Applications (World Scientific, Singapore, 2014), pp. 226-251.

[51] C. Godreche, S. N. Majumdar, and G. Schehr, Record Statistics of a Strongly Correlated Time Series: Random Walks and Lévy Flights, J. Phys. A 50, 333001 (2017).

[52] D. Boyer and C. Solis-Salas, Random Walks with Preferential Relocations to Places Visited in the Past and Their Application to Biology, Phys. Rev. Lett. 112, 240601 (2014).

[53] A. Falcón-Cortés, D. Boyer, L. Giuggioli, and S. N. Majumdar, Localization Transition Induced by Learning in Random Searches, Phys. Rev. Lett. 119, 140603 (2017).

[54] W. Feller, An Introduction to Probability Theory and Its Applications (John Wiley \& Sons, New York, 1950), Vol. 2.

[55] F. Spitzer, Principles of Random Walk, 2nd ed. (SpringerVerlag, New York, 1976), Vol. 34.

[56] J. Rudnick and G. Gaspari, Elements of the Random Walk: An Introduction for Advanced Students and Researchers (Cambridge University Press, Cambridge, England, 2004).

[57] P. Révész, Random Walk in Random and Non-Random Environments (World Scientific, Singapore, 2005).

[58] P. Flajolet and R. Sedgewick, Analytic Combinatorics (Cambridge University Press, Cambridge, England, 2009).

[59] F. Avram and M. Vidmar, First Passage Problems for Upwards Skip-Free Random Walks via the Scale Functions Paradigm, Adv. Appl. Probab. 51, 408 (2019).

[60] A. Bostan, Computer Algebra for Lattice Path Combinatorics. Habilitation, Université Paris 13, Laboratoire d'Informatique de Paris Nord, 2017.

[61] The 1D solution was apparently already known by Abraham de Moivre and Pierre-Simon Laplace, as reported in Ref. [54], and by Jan Cornelis Kluyvert, as reported in Refs. [9,62].

[62] B Carazza, The History of the Random-Walk Problem: Considerations on the Interdisciplinarity in Modern Physics, Riv. Nuovo Cimento 7, 419 (1977).

[63] S. Karlin and J. McGregor, Random Walks, Illinois J. Math. 3, 66 (1959).

[64] W. Schoutens, Stochastic Processes and Orthogonal Polynomials (Springer, New York, 2012).

[65] F. A. Grünbaum, Random Walks and Orthogonal Polynomials: Some Challenges, in Probability, Geometry and Integrable Systems, edited by M. Pinsky and B. Birnir (Cambridge University Press, Cambridge, England, 2007), pages 241-260.

[66] E.W. Montroll, Random Walks in Multidimensional Spaces, Especially on Periodic Lattices, SIAM J. Appl. Math. 4, 241 (1956).

[67] Z. Kalay, Effects of Confinement on the Statistics of Encounter Times: Exact Analytical Results for Random Walks in a Partitioned Lattice, J. Phys. A 45, 215001 (2012).

[68] D. A. Levin and Y. Peres, Markov Chains and Mixing Times, 2nd ed. (American Mathematical Society, Providence, 2017).

[69] M. N. Barber and B. W. Ninham, Random and Restricted Walks: Theory and Applications (Gordon \& Breach, New York, 1970).
[70] M. Sahimi, B. D. Hughes, L. E. Scriven, and H. T. Davis, On Pólya Random Walks, Lattice Green Functions, and the Bond Percolation Threshold, J. Phys. A 16, L67 (1983).

[71] K. Lakatos-Lindenberg, R. P. Hemenger, and R. M. Pearlstein, Solutions of Master Equations and Related Random Walks on Quenched Linear Chains, J. Chem. Phys. 56, 4852 (1972).

[72] J. Abate, G. L. Choudhury, and W. Whitt, An Introduction to Numerical Transform Inversion and Its Application to Probability Models, in Computational Probability, edited by W. K. Grassman (Kluwer, Boston, 1999), pp. 257-323.

[73] J.-H. Yoon and H. Kim, Exact Fundamental Functions of the Random Walk in One-Dimensional Confined System, Bull. Korean Chem. Soc. 38, 364 (2017).

[74] W.-C. Yueh and S.S. Cheng, Explicit Eigenvalues and Inverses of Tridiagonal Toeplitz. Matrices with Four Perturbed Corners, ANZIAM J. 49, 361 (2008).

[75] See Supplemental Material at http://link.aps.org/ supplemental/10.1103/PhysRevX.10.021045 for further details on the derivations.

[76] S. A. Rice, Diffusion-Limited Reactions (Elsevier, Amsterdam, 1985), Vol. 25.

[77] T. Antal and S. Redner, Escape of a Uniform Random Walk from an Interval, J. Stat. Phys. 123, 1129 (2006).

[78] T. Odagaki and M. Lax, ac Hopping Conductivity of a One-Dimensional Bond-Percolation Model, Phys. Rev. Lett. 45, 847 (1980).

[79] R. M. Mazo, On the Green's Function for a OneDimensional Random Walk, Cell Biophysics 11, 19 (1987).

[80] H. S. Carslaw and J. C. Jaeger, Conduction of Heat in Solids, 2nd ed. (Clarendon Press, Oxford, 1959).

[81] P. M. Morse and H. Feshbach, Methods of Theoretical Physics, Part I (McGraw-Hill, New York, 1953).

[82] A. D. Polyanin, Handbook of Linear Partial Differential Equations for Engineers and Scientists (CRC Press, Boca Raton, 2002).

[83] V. M. Kenkre, The Master Equation Approach: Coherence, Energy Transfer, Annihilation, and Relaxation, in Exciton Dynamics in Molecular Crystals and Aggregates, edited by G. Hoehler (Springer, New York, 1982), pp. 1-109.

[84] V. M. Kenkre, L. Giuggioli, and Z. Kalay, Molecular Motion in Cell Membranes: Analytic Study of FenceHindered Random Walks, Phys. Rev. E 77, 051907 (2008).

[85] V. M. Kenkre and P. E. Parris, Exciton Trapping and Sensitized Luminescence: A Generalized Theory for All Trap Concentrations, Phys. Rev. B 27, 3221 (1983).

[86] V. M. Kenkre, P. E. Parris, and S. M. Phatak, Motion and Capture of Quasiparticles in Solids in the Presence of Cooperative Trap Interactions, Physica A 128, 571 (1984).

[87] P. E. Parris, S. M. Phatak, and V. M. Kenkre, Motion and Capture in the Presence of Cooperative Trap Interactions II: Exact Calculations for Perfect Absorbers in One Dimension, J. Stat. Phys. 35, 749 (1984).

[88] T. G. Mattos, C. Mejía-Monasterio, R. Metzler, and G. Oshanin, First Passages in Bounded Domains: When Is the 
Mean First Passage Time Meaningful?, Phys. Rev. E 86, 031143 (2012).

[89] A. Godec and R. Metzler, Universal Proximity Effect in Target Search Kinetics in the Few-Encounter Limit, Phys. Rev. X 6, 041037 (2016).

[90] V. M. Kenkre and D. Schmid, Comments on the Exciton Annihilation Constant and the Energy Transfer Rate in Naphthalene and Anthracene, Chem. Phys. Lett. 94, 603 (1983).

[91] V. M. Kenkre, P. E. Parris, and D. Schmid, Investigation of the Appropriateness of Sensitized Luminescence to Determine Exciton Motion Parameters in Pure Molecular Crystals, Phys. Rev. B 32, 4946 (1985).

[92] D. S. Grebenkov, R. Metzler, and G. Oshanin, Strong Defocusing of Molecular Reaction Times Results from an Interplay of Geometry and Reaction Control, Commun. Chem. 1, 96 (2018).

[93] E. W. Montroll and R. B. Potts, Effect of Defects on Lattice Vibrations, Phys. Rev. 100, 525 (1955).

[94] K. Spendier and V. M. Kenkre, Analytic Solutions for Some Reaction-Diffusion Scenarios, J. Phys. Chem. B 117, 15639 (2013).

[95] D. Hartich and A. Godec, Duality between Relaxation and First Passage in Reversible Markov Dynamics: Rugged Energy Landscapes Disentangled, New J. Phys. 20, 112002 (2018).

[96] D. Hartich and A. Godec, Extreme Value Statistics of Ergodic Markov Processes from First Passage Times in the Large Deviation Limit, J. Phys. A 52, 244001 (2019).

[97] S. Condamin, O. Bénichou, and M. Moreau, First-Passage Times for Random Walks in Bounded Domains, Phys. Rev. Lett. 95, 260601 (2005).

[98] S Condamin and O Bénichou, Exact Expressions of Mean First-Passage Times and Splitting Probabilities for Random Walks in Bounded Rectangular Domains, J. Chem. Phys. 124, 206103 (2006).

[99] S. Condamin, O. Bénichou, and M. Moreau, Random Walks and Brownian Motion: A Method of Computation for First-Passage Times and Related Quantities in Confined Geometries, Phys. Rev. E 75, 021111 (2007).

[100] H. Larralde and G. H. Weiss, A Generating Function for the Second Moment of the Distinct Number of Sites Visited by an n-step Lattice Random Walk, J. Phys. A 28, 5217 (1995).

[101] T. Calandre, O. Bénichou, D. S. Grebenkov, and R. Voituriez, Splitting Probabilities and Interfacial Territory Covered by Two-Dimensional and Three-Dimensional Surface-Mediated Diffusion, Phys. Rev. E 89, 012149 (2014).

[102] T. Weng, Ji. Zhang, M. Small, J. Yang, F. H. Bijarbooneh, and P. Hui, Multitarget Search on Complex Networks: A Logarithmic Growth of Global Mean Random Cover Time, Chaos 27, 093103 (2017).

[103] C. Chevalier, O. Bénichou, B. Meyer, and R. Voituriez, First-Passage Quantities of Brownian Motion in a Bounded Domain with Multiple Targets: A Unified Approach, J. Phys. A 44, 025002 (2011).

[104] I. Majid, D. Ben-Avraham, S. Havlin, and H. E. Stanley, Exact-Enumeration Approach to Random Walks on Percolation Clusters in Two Dimensions, Phys. Rev. B 30, 1626 (1984).
[105] S. Baghram, F. Nikakhtar, M. R. R. Tabar, S. Rahvar, R. K. Sheth, K. Lehnertz, and M. Sahimi, Exact Enumeration Approach to First-Passage Time Distribution of NonMarkov Random Walks, Phys. Rev. E 99, 062101 (2019).

[106] N. Masuda, M. A. Porter, and R. Lambiotte, Random Walks and Diffusion on Networks, Phys. Rep. 716, 1 (2017).

[107] M. H. Ernst, Random Walks with Short Memory, J. Stat. Phys. 53, 191 (1988).

[108] V. Tejedor, R. Voituriez, and O. Bénichou, Optimizing Persistent Random Searches, Phys. Rev. Lett. 108, 088103 (2012).

[109] L. Giuggioli, I. Arye, A. H. Robles, and G. A. Kaminka, From Ants to Birds: A Novel Bio-Inspired Approach to Online Area Coverage, in Distributed Autonomous Robotic Systems (Springer, New York, 2018), pp. 31-43.

[110] E. W. Montroll, Stochastic Processes and Chemical Kinetics, in Energetics in Metallurgical Phenomena (Gordon and Breach, New York, 1967), Vol. 3, pp. 125-187.

[111] V. M. Kenkre, Z. Kalay, and P. E. Parris, Extensions of Effective-Medium Theory of Transport in Disordered Systems, Phys. Rev. E 79, 011114 (2009).

[112] V. M. Kenkre, E. W. Montroll, and M. F. Shlesinger, Generalized Master Equations for Continuous-Time Random Walks, J. Stat. Phys. 9, 45 (1973).

[113] V. M. Kenkre, The Generalized Master Equation and Its Applications, in Statistical Mechanics and Statistical Methods in Theory and Application, edited by U. Landman (Plenum, New York, 1977), pp. 441-461.

[114] A. Comtet and S. N. Majumdar, Precise Asymptotics for a Random Walker's Maximum, J. Stat. Mech. (2005) P06013.

[115] C. Banderier and P. Flajolet, Basic Analytic Combinatorics of Directed Lattice Paths, Theor. Comput. Sci. 281, 37 (2002).

[116] S. N. Majumdar and R. M. Ziff, Universal Record Statistics of Random Walks and Lévy Flights, Phys. Rev. Lett. 101, 050601 (2008).

[117] S. N. Majumdar, G. Schehr, and G. Wergen, Record Statistics and Persistence for a Random Walk with a Drift, J. Phys. A 45, 355002 (2012).

[118] M. R. Evans and S. N. Majumdar, Diffusion with Stochastic Resetting, Phys. Rev. Lett. 106, 160601 (2011).

[119] M. R. Evans and S. N. Majumdar, Diffusion with Resetting in Arbitrary Spatial Dimension, J. Phys. A 47, 285001 (2014).

[120] A. Nagar and S. Gupta, Diffusion with Stochastic Resetting at Power-Law Times, Phys. Rev. E 93, 060102 (2016).

[121] S. Gupta and A. Nagar, Resetting of Fluctuating Interfaces at Power-Law Times, J. Phys. A 49, 445001 (2016).

[122] M. R. Evans, S. N. Majumdar, and G. Schehr, Stochastic Resetting and Applications, arXiv:1910.07993.

[123] G. F. Lawler, Loop-Erased Random Walk, in Perplexing Problems in Probability (Birkhäuser, Boston, 1999), pp. 197-217.

[124] D. Boyer, A. Falcón-Cortés, L. Giuggioli, and S. N. Majumdar, Anderson-Like Localization Transition of Random Walks with Resetting, J. Stat. Mech. (2019) 053204.

[125] S. Condamin, V. Tejedor, and O. Bénichou, Occupation Times of Random Walks in Confined Geometries: From 
Random Trap Model to Diffusion-Limited Reactions, Phys. Rev. E 76, 050102 (2007).

[126] O. Bénichou and R. Voituriez, Narrow-Escape Time Problem: Time Needed for a Particle to Exit a Confining Domain through a Small Window, Phys. Rev. Lett. 100, 168105 (2008).

[127] D. Holcman and Z. Schuss, The Narrow Escape Problem, SIAM Rev. 56, 213 (2014).

[128] D. S. Grebenkov and J.-F. Rupprecht, The Escape Problem for Mortal Walkers, J. Chem. Phys. 146, 084106 (2017).

[129] S. N. Majumdar, S. Sabhapandit, and G. Schehr, Exact Distributions of Cover Times for $N$ Independent Random Walkers in One Dimension, Phys. Rev. E 94, 062131 (2016).

[130] M. Chupeau, O. Bénichou, and R. Voituriez, Cover Times of Random Searches, Nat. Phys. 11, 844 (2015).

[131] V. Tejedor, M. Schad, O. Bénichou, R. Voituriez, and R. Metzler, Encounter Distribution of Two Random Walkers on a Finite One-Dimensional Interval, J. Phys. A 44, 395005 (2011).

[132] L. Giuggioli, S. Pérez-Becker, and D. P. Sanders, Encounter Times in Overlapping Domains: Application to Epidemic Spread in a Population of Territorial Animals, Phys. Rev. Lett. 110, 058103 (2013).

[133] L. Giuggioli, G. Abramson, V. M. Kenkre, R. R. Parmenter, and T.L. Yates, Theory of Home Range Estimation from Displacement Measurements of Animal Populations, J. Theor. Biol. 240, 126 (2006).

[134] V. M. Kenkre and S Sugaya, Theory of the Transmission of Infection in the Spread of Epidemics: Interacting Random Walkers with and without Confinement, Bull. Math. Biol. 76, 3016 (2014).

[135] S. Sugaya and V. M. Kenkre, Analysis of Transmission of Infection in Epidemics: Confined Random Walkers in Dimensions Higher than One, Bull. Math. Biol. 80, 3106 (2018).

[136] L. Lizana and T. Ambjörnsson, Single-File Diffusion in a Box, Phys. Rev. Lett. 100, 200601 (2008).

[137] L. Lizana and T. Ambjörnsson, Diffusion of Finite-Sized Hard-Core Interacting Particles in a One-Dimensional Box: Tagged Particle Dynamics, Phys. Rev. E 80, 051103 (2009).

[138] M. F. Shlesinger, G. M. Zaslavsky, and J. Klafter, Strange Kinetics, Nature (London) 363, 31 (1993).

[139] G. Zumofen, J. Klafter, and M. F. Shlesinger, Lévy Flights and Lévy Walks Revisited, in Anomalous Diffusion from Basics to Applications (Springer, New York, 1999), pp. $15-34$.

[140] J. Klafter and I. M. Sokolov, First Steps in Random Walks: From Tools to Applications (Oxford University, New York, 2011).
[141] M.F. Shlesinger, Origins and Applications of the Montroll-Weiss Continuous Time Random Walk, Eur. Phys. J. B 90, 93 (2017).

[142] G. Bel and E. Barkai, Weak Ergodicity Breaking in the Continuous-Time Random Walk, Phys. Rev. Lett. 94, 240602 (2005).

[143] G. Bel and E. Barkai, Random Walk to a Nonergodic Equilibrium Concept, Phys. Rev. E 73, 016125 (2006).

[144] E. Barkai, R. Metzler, and J. Klafter, From Continuous Time Random Walks to the Fractional Fokker-Planck Equation, Phys. Rev. E 61, 132 (2000).

[145] I. M. Sokolov, A. Blumen, and J. Klafter, Linear Response in Complex Systems: CTRW and the Fractional FokkerPlanck Equations, Physica A 302, 268 (2001).

[146] R. Pemantle and M. C. Wilson, Analytic Combinatorics in Several Variables (Cambridge University Press, Cambridge, England, 2013), Vol. 140.

[147] J. C. Mason and D. C. Handscomb, Chebyshev Polynomials (Chapman and Hall/CRC, Boca Raton, 2002).

[148] F. W. J Olver, D. W. Lozier, R. F. Boisvert, and C. W. Clark, NIST Handbook of Mathematical Functions (Cambridge University Press, Cambridge, England, 2010).

[149] B. C. Berndt and B. P. Yeap, Explicit Evaluations and Reciprocity Theorems for Finite Trigonometric Sums, Adv. Appl. Math. 29, 358 (2002).

[150] X. Wang and D.-Y. Zheng, Summation Formulae on Trigonometric Functions, J. Math. Anal. Appl. 335, 1020 (2007).

[151] W. Chu, Partial Fraction Decompositions and Trigonometric Sum Identities, Proc. Am. Math. Soc. 136, 229 (2008).

[152] M. Beck and M. Halloran, Finite Trigonometric Character Sums via Discrete Fourier Analysis, Int. J. Number Theory, 6, 51 (2010).

[153] C. M. Da Fonseca, M. L. Glasser, and V. Kowalenko, Basic Trigonometric Power Sums with Applications, Ramanujan J. 42, 401 (2017).

[154] S. R. Holcombe, Falling Coupled Oscillators and Trigonometric Sums, Z. Angew. Math. Phys. 69, 19 (2018).

[155] I. S. Gradshteyn and I. M. Ryzhik, Table of Integrals, Series, and Products (Academic Press, San Diego, 2014).

[156] K. Aghigh, M. Masjed-Jamei, and M. Dehghan, A Survey on Third and Fourth Kind of Chebyshev Polynomials and Their Applications, Appl. Math. Comput. 199, 2 (2008).

[157] E. R. Hansen, A Table of Series and Products (PrenticeHall, Englewood Cliffs, NJ, 1975).

[158] D. Zill and P. Shanahan, A First Course in Complex Analysis with Applications (Jones \& Bartlett Publishers, Sudbury, MA, 2009). 